\title{
Spiders (Aranei) of the typical tundra subzone of the Yugorsky Peninsula, Russia
}

\section{Пауки (Aranei) типичных тундр Югорского полуострова, Россия}

\author{
Andrei V. Tanasevitch, Olga A. Khruleva \\ А.В. Танасевич, О.А. Хрулёва
}

\begin{abstract}
A.N. Severtsov Institute of Ecology and Evolution, Russian Academy of Sciences, Leninsky prospekt 33, Moscow 119071, Russia. E-mail: tanasevitch@gmail.com; oa-khruleva@mail.ru

Институт проблем экологии и эволюции имени А.Н. Северцова РАН, Ленинский проспект 33, Москва 119071, Россия.
\end{abstract}

KEY WORDS: Russian Arctic, tundra, chorology, checklist, Amderma, Nenets A.O., habitat and stratification spreading.

КЛЮЧЕВЫЕ СЛОВА: Российская Арктика, тундра, хорология, список видов, Амдерма, НАО, биотопическое и ярусное распределение.

ABSTRACT. An araneofauna of the vicinity of Amderma village in the northern part of the Yugorsky Peninsula, the subzone of typical tundra of the tundra zone, has been studied and analyzed. Some peculiarities of the spider fauna and population, as well as distributional patterns of species and chorological groups across the main zonal-landscape types of vegetation, have been established. The local fauna consists of 53 spider species in seven families, its taxonomic composition has oligotaxon pattern and can be described as Lin(85)-Lyc(4)-Tho(4). Compared to other local spider faunas of typical Eurasian tundras, the studied one differs in a lower proportion of Lycosidae. The linyphiid species Islandiana cristata Eskov, 1987 and Proislandiana pallida (Kulczyński, 1908) have been recorded from the European fauna for the first time. The studied araneofauna can be characterized as Siberian, with a great share of widespread species and a small proportion of European and East Palaearctic ones. A share of the arctic fraction (i.e., arctic, arctoalpine and arcto-montane species) is $53 \%$, that of hypoarctic elements (i.e., hypoarctic, hypoarctic-montane and hypoarctic-boreo-montane species) - 30\%, the boreal and polyzonal fractions account for $8 \%$ and $9 \%$, respectively. The diversity of spider assemblages of the main vegetation types are as follows: 48 species in intrazonal communities, 29 in anthropogenic, 17 in zonal and 12 in azonal. Zonal tundras are distinct by a large share of the arctic fraction (76\%), with boreal species being absent. A river valley is the source (or refugium) of "southern" elements, considerably increasing their share in the fauna of local tundras. Some of these species are restricted to the river valley only, although others can come out to the watershed and colonize its intrazonal and/or anthropogenic habitats. Species of the arctic fraction inhabit a wider range of tundra vegetation types, as compared to spiders of other fractions, but only a small their part is confined to zonal tundras.
A large part of representatives of the arctic fraction is absent from zonal habitats and tend to occur in intrazonal biotopes of the watershed and/or river valley: viz., purely arctic species markedly prefer wet intrazonal habitats, whereas arcto-montane and arcto-alpine ones occur in the areas with gravelly soil. Species of the boreal fraction are absent from most habitats, congregating in wet intrazonal biotopes, the river valley and anthropogenic habitats. Most species and the vast majority of collected specimens were found in the ground (epigeal) layer. Only a few rare species were associated with the herbaceous layer: e.g., some abundant Semljicola species were recorded from there in certain days with favorable weather conditions. Embolic division of Erigone longipalpis (Sundevall, 1830) and E. tirolensis L. Koch, 1872 are illustrated.

How to cite this article: Tanasevitch A.V., Khruleva O.A. 2017. Spiders (Aranei) of the typical tundra subzone of the Yugorsky Peninsula, Russia // Arthropoda Selecta. Vol.26. No.4. P.341-368. doi: 10.15298/ arthsel.26.4.10

РЕЗЮМЕ. Проведён анализ аранеофауны окрестностей пос. Амдерма, расположенного в северной части Югорского полуострова, в подзоне типичных тундр тундровой зоны. Выявлены особенности фауны и населения пауков, а также характер распределения видов и хорологических групп по основным зонально-ландшафтным типам растительности. Локальная фауна насчитывает 53 вида семи семейств, имеет олиготаксонный облик, описываемый формулой Lin(85)-Lyc(4)-Tho(4). Среди прочих локальных фаун типичных тундр Евразии её отличает более низкий удельный вес Lycosidae. Виды Islandiana cristata Eskov, 1987 и Proislandiana pallida (Kulczyński, 1908) впервые отмечены в фауне Европы. По составу зоогеографических элементов аранеофауну можно охарактеризовать как си- 
бирскую, с высокой долей участия широкоареальных и малой долей европейских и восточнопалеарктических элементов. Доля арктической фракции в фауне (арктические, аркто-альпийские и аркто-монтанные виды) составляет 53\%, гипоарктической (гипоарктические, гипоаркто-монтанные и гипоарктоборео-монтанные виды) - 30\%, бореальная и полизональная фракции составляют 8 и 9\%, соответственно. По объёму фауны основные типы растительных сообществ распределились следующим образом: интразональные (48 видов), антропогенные (29), зональные (17) и азональные (12). Зональные тундры лидируют по доле видов арктической фракции (76\%), элементы бореальной фракции здесь отсутствуют. Долина реки играет роль поставщика (или рефугиума) «южных» элементов, существенно увеличивая их долю в фауне местных тундр. Часть этих видов ограничена в своём распространении лишь долиной реки, однако некоторые виды вышли на водораздел, где заселили интразональные и(или) антропогенные местообитания. Виды арктической фракции осваивают более широкий топический диапазон, чем виды других фракций, но лишь небольшая их часть демонстрирует приуроченность к зональным тундрам. Бо́льшая часть видов арктической фракции отсутствует в 3ональных местообитаниях, заселяя преимущественно интразональные биотопы на водоразделе и(или) в долине реки, причём, чисто арктические виды предпочитают преимущественно сырые интразональные местообитания, тогда как аркто-монтанные и аркто-альпийские виды явно тяготеют к участкам с щебнистыми грунтами. Виды бореальной фракции демонстрируют наиболее узкий топический диапазон, концентрируясь в сырых биотопах речной долины и в антропогене. Основная часть видов и подавляющее большинство экземпляров пауков собранны в наземном ярусе. К травостою приурочено лишь несколько редких в местных тундрах видов; некоторые массовые виды рода Semljicola отмечены в травостое в отдельные дни с благоприятными погодными условиями. Приведены рисунки эмболюсного отдела Erigone longipalpis (Sundevall, 1830) и E. tirolensis L. Koch, 1872.

\section{Introduction}

Tundra zone of Europe spread for almost 1,500 km, embracing a narrow belt of the Far North of the Kola Peninsula and rather vast space of North Russian Plain. The spider fauna of tundra of the Kola Peninsula were concerned by a paper of Tanasevitch \& Rybalov [2010], which briefly reported the history of studies and analyzed the local tundra araneofauna including 105 spider species. The publication of Nekhaeva \& Nekhaev [2011] added one more species to the list.

Tundras of the Russian Plain occupy the territory from the Kanin Peninsula in the West to far northern branches of the Polar Urals (Pai-Khoi Ridge) in the
East; in general, araneofauna of this natural region is rather well studied. Initial information about spiders of continental tundra appeared in the publication of Pokrovsky [1904], where the author lists two species of spiders from southern tundra of the Kanin Peninsula. Eight species of spiders were mentioned by Kulczyński [1916] from lower reaches of the Kara River and the Kara tundra (see below). The next paper on the tundra spiders was published only 70 years later [Eskov, 1985], it contained data on records of 17 spider species from Yugorsky Peninsula. In subsequent several years, a series of taxonomic articles has been published, with description of new spider species from tundras of the Russian Plain [Eskov, 1981, 1986, 1987; 1988a, b; Tanasevitch, 1982, 1984a, b, 2000; Tanasevitch, Eskov, 1987, etc.]. After these publications, the first papers were released that were specially devoted to the fauna and biotopic distribution of spiders of European tundras. Of them, the first ones [Mazura, 2000; Mazura, Esyunin, 2001] dealt with spiders of the Pechora River delta in the Malozemelskaya tundra. The authors have recorded 116 species and gave the first data on their chorology. Several years later all the known information and supplemented with a lot of new data on spiders of European tundras was summarized by Tanasevitch \& Koponen [2007]. In that paper, the authors gave a complete faunal list of spiders of southern tundra of the Russian Plain (216 species), with a detailed zoogeographic analysis, and also gave an explicit picture of the zonal-landscape distribution of the spiders. The recent work on soil invertebrates of the Bolshezemelskaya tundra [Taskaeva et al., 2015] did not supplement the species list. At the moment, the spider fauna of continental tundra, including coastal tundra of the Kola Peninsula, numbers 243 species (14 families).

The study of araneofauna of arctic islands from the European Russian sector has started in XIX century, and its history was in detail described by Tanasevitch [2017c]. At present, one species is known from Kolguev Island [Tanasevitch, 2017b], 52 species — from Dolgy Island [Marusik et al., 2016]; 20 and 21 species from Vaygach Island and Novaya Zemlya Archipelago, respectively [Tanasevitch, 2017c]; the araneofauna of polar deserts of Franz Josef Land Archipelago contains only two species [Bulavintsev, Babenko, 1983].

In total, the insular araneofauna of the European Russian sector of the Arctic contains 63 species (6 families), and the whole fauna of spiders of the continental tundras of Russia lists 259 species (14 families).

The Yugorsky Peninsula, the araneofauna of which is devoted this paper, is a large peninsula in the far north-east of Europe, belonging to the Nenets Autonomous Okrug of the Arkhangelsk Area of Russia. The peninsula is washed by the Barents Sea from the West and by the Kara Sea from the East, its southern border lies along the line connecting the mouths of the Korotaikha and Kara rivers (see the map). The peninsula is situated near the border between Europe and Asia (Siberia) and its boundary position is of certain interest 


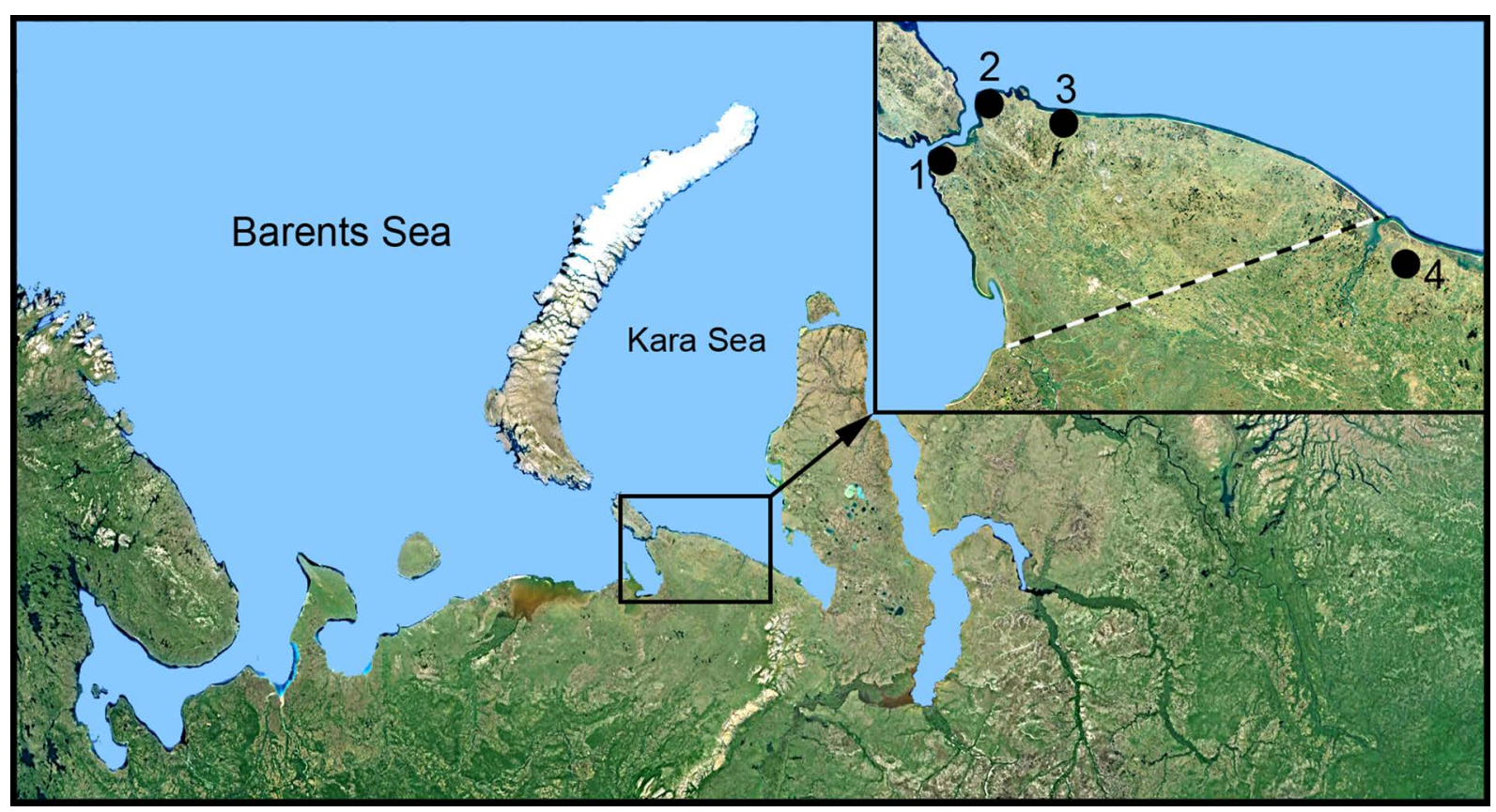

Map. Collecting localities in the Yugorsky Peninsula (1-3) and in adjacent territory (4). 1 — Cape Belyi Nos; 2 — Yugorsky Shar Polar Station; 3 - Amderma and its vicinities; 4 - Kara River and Kara tundra.

Карта. Месторасположение точек сборов на Югорском п-ове (1-3) и прилегающей территории (4). 1 -- мыс Белый Нос; 2 полярная станция Югорский Шар; 3 - Амдерма и её окрестности; 4 - р. Кара и Карская тундра.

from zoogeographical viewpoint. Information about spiders of the Yugorsky Peninsula is contained in the single faunistic paper [Eskov, 1985], where the author gave a list of spiders (17 species) collected in two localities of northern coast of the peninsula, i.e., Cape Belyi Nos and Yugorsky Shar Polar Station (see the map); two more species were added somewhat later [Eskov, 1988a, 1989]. All these species, except for Alopecosa solivaga (Kulczyński, 1901) and Tarsiphantes latithorax Strand, 1905, were found by us in the study area.

\section{Material and methods}

The present work is based on the extensive material collected by the authors in vicinities of Amderma village of Nenets Autonomous Area (see the map) in the period from July 23 to August 5, 2016. Spiders were collected by sifting moss and litter, pitfall trapping, net-sweeping and hand collecting. Pitfall traps $(200 \mathrm{ml}$ plastic cups, filled by water) were positioned in 14 studied biotopes in lines of 10-20 traps. Twelve lines of traps were positioned in plots with natural vegetation, 2 lines in 3 plots with human impact. The sampling was performed every 3-5 days. In total, 2037 trap-days have been made. Net-sweeping was used mainly on the watershed and in the village, at daytime, by 3-4 times in each biotope; 60 net-sweeping samples was undertaken. In total, 2580 adult spider specimens were gained and examined.
In the present paper, the previously used principle of the species range typification was reconsidered. Specifically, the latitudinal component was refined (i.e., arctic, boreal, etc.), whereas the regional one (i.e., European, Siberian, etc.) remained the same. Since spiders include rather many species occurring in adjacent natural zones, the priority task was to reveal that part of the latitudinal gradient which is most characteristic of the species, specifically: association of most records to it, regularity of findings in suitable biotopes, expansion of topic range, high density, etc. In doing this, we used both original and literature data. As a result of the new typological practice, the range latitudinal component of many species has been changed and became different from the previously published papers (see Tanasevitch \& Koponen [2007]; Tanasevitch \& Rybalov [2015]; Tanasevitch \& Nekhaeva [2016], etc.). Some species among widely spread in tundra but known also from some areas of taiga zone and referred earlier to arcto-boreal are considered here as arctic. Besides, one more type, hypoarctic, was added to the range of latitudinal types of distribution. This term was used by some arachnologists (see Marusik \& Eskov [2009]; Esyunin [2015], etc.) but previously we avoided to use it because we did not see a need for its usage within the framework of old scheme of typification.

Abbreviations in Figures: Co - column; D - duct; $\mathrm{E}$ - embolus; EM - embolic membrane, the membrane arising directly from the radix; EP - embolus proper; MeRT - mesal radical tooth sensu Tanasevitch [2017a] = mesal tooth sensu Crosby \& Bishop [1928]; 
MM - median membrane sensu Helsdingen [1965]; MRT - median radical tooth sensu Tanasevitch [2017a] = median tooth sensu Crosby \& Bishop [1928]; PRA - posterior radical apophysis sensu Tanasevitch [2017a] = posterior tooth sensu Crosby \& Bishop [1928]; R — radix.

\section{Latitudinal fractions}

To make the analysis easier, we united the studied species of local fauna into four latitudinal fractions (after Yurtsev et al. [2010]).

Arctic fraction (28 species). This group includes species with optimum of distribution in plain landscapes lying northward of timberline. It includes purely arctic (20 species), arcto-alpine (5) and arcto-montane species (3). The purely arctic cluster in addition comprises species with meta-arctic distribution (after Gorodkov [1984]), i.e., those which penetrate to the south along mountains only in polar and near-polar regions and only slightly pass beyond the Arctic.

Hypoarctic fraction (16 species). It contains species with optimum of distribution mostly in landscapes of southern tundra zone, forest-tundra, subzone of northern taiga (hypoarctic distribution) and - in mountains - the upper part of forest belt and the upper part of the surroundings of woodless mountain tops (hypoarcto-montane distribution). We also include in hypoarctic fraction several species with hypoarcto-borealmontane distribution, which are typical among components of hypoarctic spider faunas in various regions of the North. Hypoarctic fraction includes hypoarctic (4), hypoarcto-boreo-montane (5) and hypoarcto-montane (7) species.

Boreal fraction (4 species). It includes species sporadically penetrating into the tundra zone, usually to its marginal areas, or those rather deeply penetrating into tundra by using specific biotopes in riverbeds/ river valleys of mostly meridional direction. The boreal fraction consists of boreo-montane (2) and boreonemoral (2) species.

Polyzonal fraction (5 species). These are species with polyzonal distribution, i.e., inhabiting virtually all the zones. These elements are rather restricted in tundra landscape but some of them regularly occur in various subzones of tundra and can be considered as a full-value component of artic biota.

We use the "purely arctic" term in the text, meaning arctic species that are thus separated from other species of the arctic fraction; "southern species" unite hypoarctic, boreal and polyzonal species.

\section{Typification of vegetative communities}

The scheme proposed by Yuri I. Chernov [1978] for classifying plant communities of the Arctic has been used for the analysis of zonal-landscape distribution of tundra spiders. Based on this classification, there are three main zonal-landscape types of plant communities in the tundra: zonal, intrazonal and azonal (for more details see Tanasevitch \& Koponen [2007]; Tanasevitch \& Rybalov [2015]). Brief descriptions are given below for these types of vegetation in tundra in the vicinities of Amderma. One more type is added: anthropogenic, which progressively occupies increasingly large areas in the Arctic.

Zonal plant communities - the plant communities that are restricted to the watershed of one zone (subzone), defining the vegetation zonal face of the landscape. In zonal communities, abiotic factors are more extreme as compared to those in intrazonal communities and therefore living conditions are more adverse. In the studied area, the zonal plant associations are represented by different variants of undershrubsedge-lichen-moss tundra rare with spots of bare ground. Undershrubs are represented by dwarf Salix spp., Vaccinium vitis-idea, Dryas and Cassiope; mosses are dominated by Pleurozium, Aulacomnium and Polytrichum, the moss cover is weak, the living layer comprising only $3-5 \mathrm{~cm}$. Vegetation rather often include Astragal and Equisetum. It should be mentioned that the zonal tundra communities completely lacks dwarf birch (Betula nana), Empetrum, Rubus chamaemorus, Vaccinium uliginosum and Ledum.

Intrazonal plant communities - the plant communities that are distributed across one or several zones (subzones); the typical examples being swamps and river valley vegetation: e.g., willow stands in floodplains, meadows, rocky and/or grassy slopes, forest stands on river banks or river terraces, etc. Commonly, parts of intrazonal communities are situated on the watershed among zonal vegetation types: these are willow shrubs in depressions, shrubby lake shores, flathill peatbogs with sedge fens, or sphagnum bogs in depressions, grassy slopes of small brooks, etc. The intrazonal communities are characterized by a smoother gradient of climatic factors (especially, of temperature and humidity), making living conditions there significantly less pessimal. In the studied area, the intrazonal communities are well represented and diverse. They include slopes of hills, monadnocks, rock outcrops and rocky debris in the tundra, wetlands, zoogenic communities, etc.

A special kind of intrazonal communities, we believe to be the Amderma River Valley, with its peculiar biotopes playing a significant role in formation of the current face of the fauna of typical tundra.

Azonal communities - the plant communities that are not confined to a certain zone (subzone) but distributed across many zones (subzones); in the studied area; this type is only represented by sea beach and ground bluffs above beach of the Kara Sea.

Anthropogenic communities - it is anthropogenically changed natural vegetation in some tundra areas, meadow formations, dump sites, dustbins and destroyed constructions in the village, etc.

It should be noted that the presented analysis of species distribution in the zonal-landscape types of vegetation is based only on the collecting efforts of the 
authors conducted in Amderma in 2016, because, regretfully, the literature data on spiders of the Yugorsky Peninsula [Eskov, 1985; 1987; 1988a, 1989] do not contain any ecological information.

\section{A brief geobotanical characteristic of the} region

According to geobotanical regionalization, the study area (far north-east of the Yugorsky Peninsula) lies in the subzone of typical tundra of European-West Siberian tundra province and belongs to the northern PaiKhoi region of Cis-Ural Ural subprovince [Lavrinenko, 2013]. Macrorelief of local tundra represents an uneven moraine plain, locally with rock outcrops, with remnant of rocky banks of the former river beds, as well as rock outliers, monadnocks, which are the northernmost extremities of the strongly decayed Hercian mountain ridge Pai-Khoi. Vegetation is rather heterogeneous due to relatively strong irregularity of mesorelief and on the plain (comparatively flat areas on the watershed) it is represented by rather monotonous zonal (see below) tundra communities. Microrelief of zonal tundra is relatively even, rarely small-hummocky, the vegetation mainly consists of dwarf willow-sedgelichen-moss tundra. It should be noted that such common for tundra undershrubs and shrubs as Arctous, Empetrum, Rubus chamaemorus, Vaccinium uliginosum and Ledum are absent in studied zonal tundra, they are common only in cloudberry flat-hill peatbogs. The dwarf birch (Betula nana) was not found by us in any of studied communities. The relatively dry slopes of flat hills are occupied by dwarf Salix-Dryas-Astragal associations. Relief depressions, drainage beds, slopes and brook banks are wet habitats and occupied by several variants of mixed sedge, herb and moss communities, usually with shrubs of Salix glauca and $S$. lanata with height up to $40-60 \mathrm{~cm}$. Quite often the single low shrubs of these willows are also present in zonal tundra. Outcrops of native rocks, slopes and flat tops of monadnocks are covered by petrophite tundra of montane type. The Amderma River flows within the study area and enters the Kara Sea. The river valley is broad, with flat bed and steep rocky banks occupied mainly by dwarf willow-Dryas communities. The river flood-plain is filled by willow thickets, moss and sedgeherb wet communities. A detailed description of the studied vegetative communities is given below.

Tundra of Amderma vicinities show distinct signs of anthropogenic impact, expressed in meadow formation in some areas of tundra, as well as in trampling down and littering of vast areas. However, the stress on natural communities considerably diminished in the last 25 years due to depopulation of the village and a drop in economic activity, which has led to onset of succession processes.

\section{Climatic peculiarities of the summer sea-} son of 2016

Summer of 2016 was abnormally warm as compared to previous years: untypical for this Arctic region hot and dry weather lasted for 1.5 months (June to mid-July), and only a few days before our field studies the local tundra were poured with rain. Dry regime and high temperatures have led to drying of moss cover and to some shrinkage of the area of wet biotopes. Certainly, this could not but affect the condition of spider population living mostly in moss layer and in wet biotopes. At the same time, the vast majority of collected spiders appeared to be adult, and our personal field experience allow to note that the very small percentage of juvenile specimens in the tundra spider population is not typical for the second third of summer season (see below).

\section{Description of types of studied biotopes and plant communities}

Zonal communities (Zonal in the text below)

Local zonal communities are represented by four variants of tundra which are rather similar to each other (Figs 1-4):

Undershrub-sedge-lichen-moss tundra with spots of bare ground on gentle SW-slope (Fig. 1), $69.76109^{\circ} \mathrm{N}$ $61.70187^{\circ} \mathrm{E}$ [1], $38 \mathrm{~m}$ a.s.1., with dwarf Salix spp., Vaccinium vitis-idea, rare Dryas and Cassiope; sedges; Pleurozium, Polytrichum, Aulacomnium; Cladonia; Astragal, rare Gramineae, etc., sifting and pitfall trapping, 24.VII.-5.VIII.2016.

Undershrub-lichen-moss tundra on gentle SW slope (Fig. 2), 69.76096 ${ }^{\circ} \mathrm{N} 61.69593^{\circ} \mathrm{E}$ [2], $28 \mathrm{~m}$ a.s.1., with dwarf Salix spp., Dryas, Vaccinium vitis-idea; Pleurozium, rare Aulacomnium and Polytrichum, Cladonia; rare sedges, Equisetum, Persicaria, etc., sifting and pitfall trapping, 24.VII.-5.VIII.2016.

Undershrub-sedge-lichen-moss tundra on gentle $\mathrm{S}$ slope (Fig. 3), 69.758667 $\mathrm{N} 61.70177^{\circ} \mathrm{E}$ [3], $30 \mathrm{~m}$ a.s.l., with dwarf Salix spp., Vaccinium vitis-idea; sedges; Pleurozium (90\%), rare Polytrichum, Aulacomnium and Cladonia; Astragal, etc., sifting and pitfall trapping, 24.VII.-4.VIII. 2016.

Dwarf willow-lichen-moss tundra (Fig. 4), 69. $76099^{\circ} \mathrm{N} 61.69307^{\circ} \mathrm{E}$ [4], $21 \mathrm{~m}$ a.s.l., with dwarf Salix spp., Dryas, Vaccinium vitis-idea, rare Cassiope; Pleurozium, Aulacomnium, Cladonia; Astragal, Equisetum, rare Gramineae, etc., sifting, 29.VII. \& 2.VIII.2016.

\section{Intrazonal communities on the watershed (Intra} in the text below)

Monadnocks, rock outliers and rock outcrops in tundra watershed (Figs 5-7) are the northernmost remnants of strongly decayed the Hercynian mountain ridge Pai-Khoi which is a continuation of the Urals. Monadnocks $6-10 \mathrm{~m}$ in height are mostly covered by petrophite tundra of montane type, $69.74313^{\circ} \mathrm{N} 61.56961^{\circ} \mathrm{E}$ 

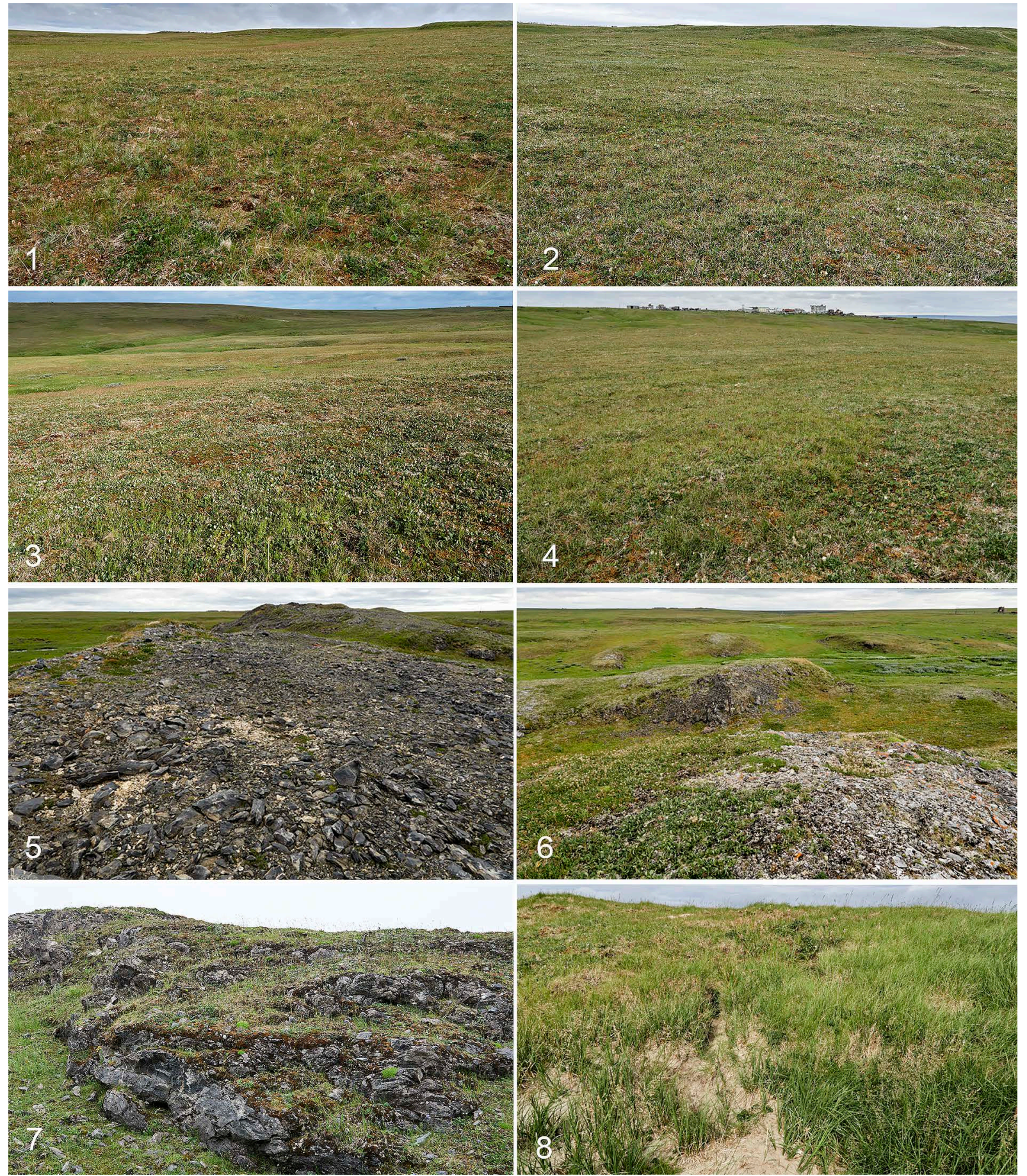

Figs 1-8. Photographs of biotopes and plant communities. 1-4 - zonal tundra; 5-7 - monadnocks, rocks, stony outliers on watershed; 8 - zoogenic community.

Рис. 1-8. Фото биотопов и растительных сообществ. 1-4 - зональная тундра; 5-7 —останцы, скалы, выходы горных пород на водоразделе; 8 - зоогенное сообщество.

[5], with dwarf Salix polaris, S. nummularia, Salix sp., Dryas, Astragal, Equisetum, Saxifraga spp.; mosses (mainly Pleurozium and Sanionia), lichens, etc., under and among stones, in tussocks of vegetation on top and slopes, sifting and hand collecting, 26.VII.2016.

Zoogenic (Fig. 8). Grass community near polar fox burrows, $69.76056^{\circ} \mathrm{N} 61.69909^{\circ} \mathrm{E}$ [6], $38 \mathrm{~m}$ a.s.1., with
Poa, Rubus chamaemorus, Equisetum, rare Bistorta, spots of Pleurozium and Cladonia, sifting and pitfall trapping, 24.VII.-5.VIII.2016.

Slopes (Figs 9-11). Tundra on slopes of flat hills.

Dry dwarf willow-Dryas-Astragal tundra on slopes to sea, $69.76578^{\circ} \mathrm{N} 61.67960^{\circ} \mathrm{E}$ [7], 8-10 $\mathrm{m}$ a.s.l., with dwarf Salix spp., Astragal, Equisetum; mosses (mainly 

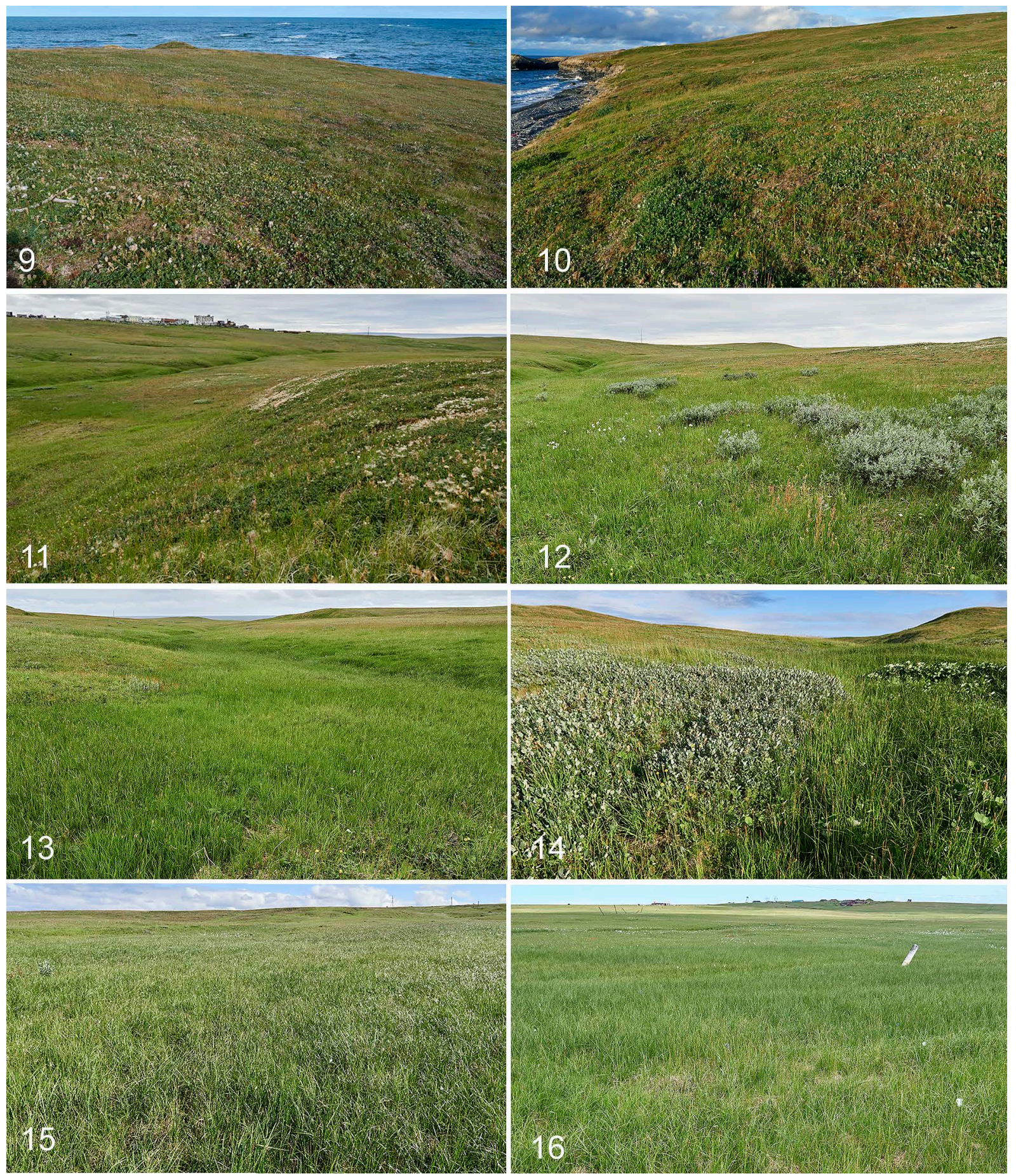

Figs 9-16. Photographs of biotopes and plant communities. 9-10 - tundra on slopes to sea (northern exposition); $11-$ tundra on slopes of flat hills on watershed (southern exposition); 12-16 - wetlands.

Рис. 9-16. Фото биотопов и растительных сообществ. 9-10 - тундра на склонах к морю (северная экспозиция); 11 - тундра на склонах увалов на водоразделе (южная экспозиция); 12-16 - сырые местообитания.

Pleurozium, Sanionia), Cladonia; rare Poa, sedges, etc., sifting and pitfall trapping, 25.VII.-5.VIII.2016.

Dry Dryas-Astragal associations on S slope of hill in tundra [8], with dwarf Salix spp., Dryas; Pleurozium, Cladonia; Astragal, etc., sifting and pitfall trapping, 24.VII.-5.VIII.2016.
Wetlands (Figs 12-16).

Willow shrubs on grassy bank of brook (Fig. 12), $69.76048^{\circ} \mathrm{N} 61.69711^{\circ} \mathrm{E}$ [9], $25 \mathrm{~m}$ a.s.l., with Salix glauca (up to $50-60 \mathrm{~cm}$ high), dwarf Salix sp.; sedges; Astragal, Equisetum, Achillea, Geranium, Chrysosplenium; thin layer of mosses, Cetraria, etc., sifting and pitfall trapping, 24.VII.-5.VIII.2016. 

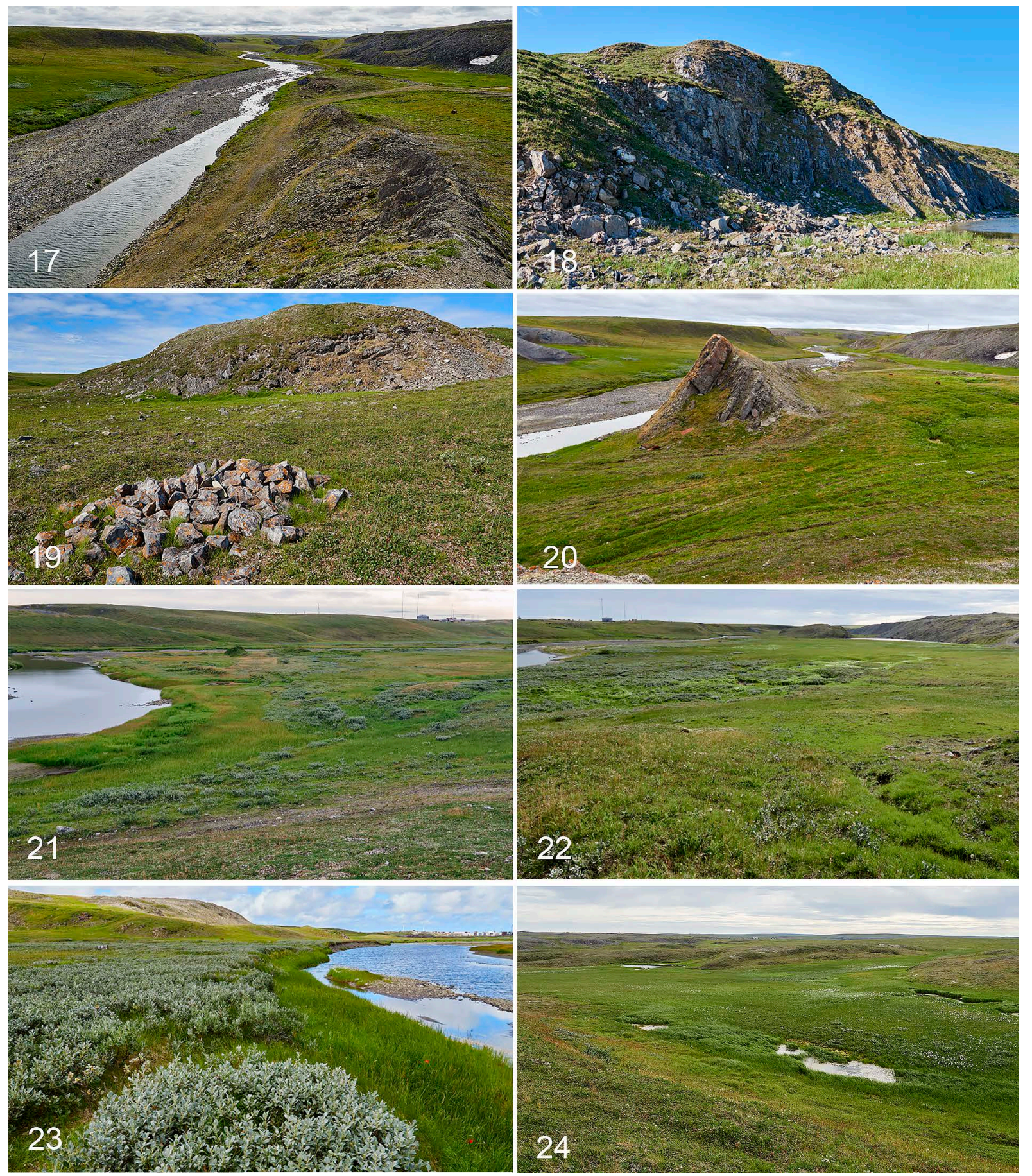

Figs 17-24. Photographs of biotopes and plant communities in riverbed of Amderma River. 17 — riverbed, overview; 18-20 — rocky river banks, stony slopes, stony debris, monadnocks; 21-24 - willow shrubs and wetlands.

Рис. 17-24. Фото биотопов и растительных сообществ в русле р. Амдерма. 17 - русло, общий вид; 18-20 - скалистые речные берега, каменистые склоны, нагромождение камней, останцы; 21-24 - ивняки и сырые местообитания.

Wet grassy flow bed in undershrub-sedge-lichenmoss tundra (Fig. 13), 69.75839 ${ }^{\circ} \mathrm{N} 61.70127^{\circ} \mathrm{E}$ [10], 28 m a.s.1., with Salix sp. shrubs; sedges, Equisetum, Poa, Comarum; thin layer of mosses (mainly Mnium), etc., sifting and pitfall traps, 24.VII.-5.VIII.2016.

Wet willow-grassy flow bed on slope to sea shore (Fig. 14), $69.76558^{\circ} \mathrm{N} 61.67989^{\circ} \mathrm{E}[11], 8 \mathrm{~m}$ a.s.l., with Salix spp. shrubs, sedges, Poa, Astragal, Equisetum; mosses (mainly Mnium), etc., sifting and pitfall trapping, 25.VII.-5.VIII.2016.

Equisetum-sedge swamp (Fig. 15), 69.76066 N 61. $68598^{\circ} \mathrm{E}$ [12], $33 \mathrm{~m}$ a.s.l., with Carex, Equisetum, Gramineae, rare Astragal; mosses (mainly Mnium, Tomentypnum), etc., sifting, 26-28.VII. \& 4.VIII.2016. 

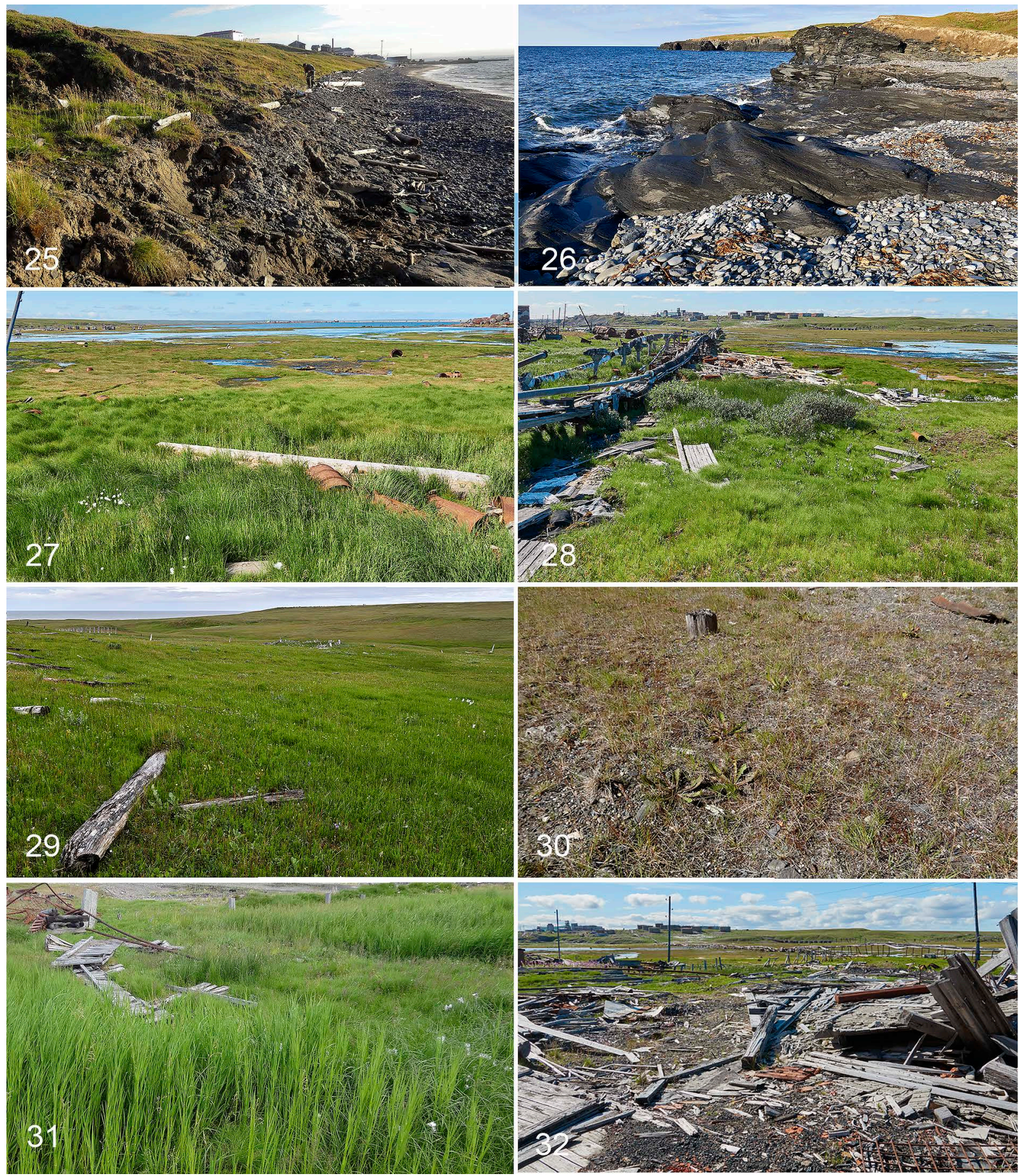

Figs 25-32. Photographs of biotopes and plant communities. 25-26 - Kara Sea shore; 27-28 — flood-plain of Amderma River near village; 29 - disturbed tundra; 30-31 — dry and wet grass communities in village, respectively; 32 - dump sites, destroyed constructions.

Рис. 25-32. Фото биотопов и растительных сообществ. 25-26 - берег Карского моря; 27-28 - пойма р. Амдерма у посёлка; 29 - антропогенно изменённая тундра; 30-31 - сухие и сырые луговые ассоциации в посёлке, соответственно; 32 - свалки, разрушенные сооружения.

Wet sedge tundra on gentle slope (Fig. 16), 69. $75591^{\circ} \mathrm{N} 61.68029^{\circ} \mathrm{E}$ [13], $34 \mathrm{~m}$ a.s.l., with dwarf Salix spp.; sedges, Poa, rare Astragal; Pleurozium, Sanionia, Tomentypnum, Mnium, etc., sifting, 30. VII.2016.
Intrazonal communities in the Amderma River Valley

Stony banks and rocky slopes (Figs 17-20). Rocky slopes, stony banks, rock outliers and outcrops, stony debris in dry bed of the Amderma River, $4 \mathrm{~km}$ up- 
stream of its mouth, $69.72752^{\circ} \mathrm{N} 61.67095^{\circ} \mathrm{E}$ [14], with dwarf willow-Dryas associations, Salix spp. (mainly $S$. reticulata), Dryas, Astragal, Saxifraga spp.; rare sedges and Equisetum; mosses (mainly Pleurozium and Sanionia), lichens, etc., under and among stones, in tussocks of vegetation, etc., sifting and hand collecting, 31.VII., 3 \& 5.VIII.2016.

Willow shrubs and wetlands in depressions (Fig. 21-24). Willow shrubs in wide riverbed of the Amderma River, $4 \mathrm{~km}$ upstream of its mouth, $69.73188^{\circ} \mathrm{N}$ $61.66235^{\circ} \mathrm{E}$ [15], $1 \mathrm{~m}$ a.s.1., with mainly Salix glauca (shrubs up to $40-60 \mathrm{~cm}$ high), rare dwarf Salix spp., Equisetum, sedges. Leaf litter practically lacks, moss layer thin, with Pleurozium, Sanionia, Aulacomnium, etc., sifting and pitfall trapping, 31.VII.-5.VIII.2016. Swampy gully, $69.72874^{\circ} \mathrm{N} 61.647089^{\circ} \mathrm{E}$ [15], with green mosses, sifting, 31.VII.2016.

Azonal communities (Azon in the text below)

Sea pebblestone beach and ground bluffs above sea beach (Figs 25-26), under stones, hand collecting and pitfall trapping [16], 25.VII.-5.VIII.2016.

Anthropogenic and anthropogenically changed tundra communities (Anthro in the text below)

Amderma River mouth, flood-plain near village (Figs 27-28) [17],with rare Salix glauca shrubs; sedges, Graminea, Equisetum; Mnium, etc., sweeping, under rubbish items, 30.VII.2016.

Disturbed tundra, dwarf willow-Equisetum tundra on gentle $\mathrm{N}$ slope, $69.75710^{\circ} \mathrm{N} 61.69843^{\circ} \mathrm{E}$ [18], $50 \mathrm{~m}$ a.s.l., with Salix spp. (mainly $S$. polaris and $S$. reticulata), Dryas, rare Cassiope, single S. glauca; Equisetum; mosses (mainly Pleurozium and Aulacomnium); rare sedges; Gramineae, Astragal, etc., sifting and pitfall trapping, 24.VII.-5.VIII.2016.

Dry and wet grass associations in village (Figs 3031) [19], sweeping, pitfall trapping, sifting, 26.VII.5.VIII.2016.

Dump sites, destroyed constructions, under rubbish items, etc. in village (Fig. 32) [20], hand collecting, 29.VII. \& 1.VIII.2016.

\section{List of spiders of the Yugorsky Peninsula}

Families are given in alphabetic order. Abbreviations Zonal, Intra, Azon and Anthro correspond to investigated types of plant communities, numbers in square brackets correspond to numbers of certain abovedescribed biotopes. Collectors: Andrei V.Tanasevitch and Olga A. Khruleva.

Fam. Araneidae

\section{Larinioides cornutus (Clerck, 1758)}

1985 Araneus cornutus. - Eskov: 127.

MATERIAL. Intra. Monadnocks: 1 juv., [5], hand collecting and sifting, 26.VII.2016. Slopes: $1 \sigma^{7}$, dry dwarf willows-DryasAstragal tundra on slopes to sea [7], sifting, 24.VII.-5.VIII.2016.
Wetlands: 1 juv., Equisetum-sedge swamp [12], sweeping, 28.VII.2016. Amderma River Valley: 1 juv., rocky slopes and rock outcrops in dry river bed [14], sifting and hand collecting, 31.VII.2016.

Anthro. 1 juv., grass associations in village [19], sweeping, 26.VII.2016.

REMARKS. In the Yugorsky Peninsula, this species has been recorded from Yugorsky Shar Polar Station [Eskov, 1985, as Araneus c.].

RANGE. Holarctic polyzonal.

Fam. Dictynidae

Dictyna major Menge, 1869

MATERIAL. Anthro. $1+$, grass associations in village [19], sweeping, 30.VII.2016.

REMARKS. This species is new to the fauna of the Yugorsky Peninsula.

RANGE. Holarctic polyzonal.

Fam. Linyphiidae

Agyneta nigripes (Simon, 1884)

MATERIAL. Intra. Monadnocks: $8 \sigma^{\top} \sigma^{7}, 6$ 우, [5], hand collecting and sifting, 26.VII.2016. Slopes: $2 \sigma^{\top} \sigma^{\top}, 5$ OO, dry dwarf willows-Dryas-Astragal tundra on slopes to sea [7], sifting and pitfall trapping, 24.VII.-5.VIII.2016. Amderma River Valley: 7 $\sigma^{\top} \sigma^{\top}, 7$ 우, rocky slopes and rock outcrops in dry river bed [14], sifting and hand collecting, 31.VII., 3 \& 5.VIII.2016.

Azon. $6 \sigma^{\top} \sigma^{\top}, 12$ \%, sea beach, under stones [16], hand collecting, 25.VIII.2016.

Anthro. $17 \sigma^{7} \sigma^{7}, 15$ 우, Amderma River mouth near village [17], flood-plain, under rubbish items, 30.VII.2016; $10 \sigma^{\top} \sigma^{\top}, 32$ 우, dump sites, destroyed constructions, under rubbish items, etc. in village [20], hand collecting, 29.VII. \& 1.VIII.2016.

REMARKS. This species is new to the fauna of the Yugorsky Peninsula.

RANGE. Holarctic arcto-alpine.

\section{Agyneta similis (Kulczyński, 1926)}

MATERIAL. Intra. Amderma River Valley: $1 \sigma^{7}, 1$, willow shrubs in flood-plain [15], sifting and pitfall trapping, 31.VII.5.VIII. 2016

REMARKS. This species is new to the fauna of the Yugorsky Peninsula.

RANGE. Fennoscandian-Siberian hypoarctic.

\section{Allomengea scopigera (Grube, 1859)}

MATERIAL. Intra. Wetlands: 1 , wet willow-grassy flow bed on slope to sea shore [11], pitfall trapping, 25-28.VII.2016; 1 $\sigma^{7}, 1$, same locality, sifting and pitfall trapping, 24.VII.5.VIII.2016. Amderma River Valley: $1 \sigma^{\top}, 1+$, rocky slopes and rock outcrops in dry river bed [14], sifting and hand collecting, 31.VII., 3 \& 5.VIII.2016; $5 \sigma^{\top} \sigma^{7}, 12$ of , willow shrubs in floodplain [15], sifting and pitfall trapping, 31.VII.-5.VIII.2016.

Anthro. $1 \sigma^{7}, 1$, Amderma River mouth near village [17], flood-plain, under rubbish items, 30.VII.2016; 2 우, dump sites, destroyed constructions, under rubbish items, etc. in village [20], hand collecting, 29.VII. \& 1.VIII.2016.

REMARKS. This species is new to the fauna of the Yugorsky Peninsula.

RANGE. Palaearctic-West Nearctic boreo-nemoral.

Arcterigone pilifrons (L. Koch, 1879)

1985 Acartauchenius pilifrons. — Eskov: 123. 
MATERIAL. Zonal. $1 \sigma^{\top}, 2$ 2 , undershrub-sedge-lichen-moss tundra with spots of bare ground [1], pitfall trapping, 2427.VII.2016; $8 \sigma^{7} \sigma^{7}, 27$ क्ष, same locality, sifting, 27.VII.2016; 1 + , same locality, pitfall trapping, 30.VII.-4.VIII.2016; $15 \sigma^{\top} \sigma^{\top}, 38$ $\rightarrow \circ$, undershrub-lichen-moss tundra [2], sifting and pitfall trapping, 1-4.VIII.2016.

REMARKS. In the Yugorsky Peninsula, this species has been recorded from Cape Belyi Nos [Eskov, 1985, as Acartauchenius $p$.].

RANGE. Siberian-West Nearctic arctic.

\section{Bathyphantes eumenis (L. Koch, 1879)}

MATERIAL. Intra. Monadnocks: 2 O , [5], hand collecting and sifting, 26.VII.2016. Amderma River Valley: 4 우, rocky slopes and rock outcrops in dry river bed [14], sifting and hand collecting, 31.VII., $3 \&$ 5.VIII.2016; $1 \sigma^{7}, 5$ क्ष, willow shrubs in flood-plain [15], sifting and pitfall trapping, 31.VII.-5.VIII.2016.

REMARKS. This species is new to the fauna of the Yugorsky Peninsula.

RANGE. Holarctic hypoarcto-boreo-montane.

Bathyphantes gracilis (Blackwall, 1841)

MATERIAL. Intra. Monadnocks: $1 \sigma^{\gamma}, 2$ o+, [5], hand collecting and sifting, 26.VII.2016; Slopes: 2 + , dry dwarf willowsDryas-Astragal tundra on slopes to sea [7], pitfall trapping, 28.VII.5.VIII.2016. Wetlands: 1 , wet grassy flow bed in tundra [10], pitfall trapping, 24-27.VII.2016; $1 \checkmark^{7}$, wet willow-grassy flow bed on slope to sea shore [11], sifting and pitfall trapping, 24.VII.5.VIII.2016; $1 \sigma^{7}$, same locality, sifting, 4.VIII.2016; $1 \Im^{7}$, Equisetum-sedge swamp [12], sifting, 28.VII.2016; $3 \sigma^{\top} \sigma^{7}, 5$ of , wet sedge tundra on gentle slope [13], sifting, 30.VII.2016. Amderma River Valley: $7 \sigma^{\top} \sigma^{\top}, 10$ 우, rocky slopes and rock outcrops in dry river bed [14], sifting and hand collecting, 31.VII., $3 \&$ 5.VIII.2016; $2 \Im^{7} \sigma^{7}$, willow shrubs in flood-plain [15], sifting and pitfall trapping, 31.VII.-5.VIII.2016.

Azon. $1 \Im^{7}$, sea beach, under stones [16], hand collecting, 25.VIII.2016.

Anthro. $5 \sigma^{7} \sigma^{7}, 10$ 우, Amderma River mouth near village [17], flood-plain, under rubbish items, 30.VII.2016; 1 q, dwarf willow-Equisetum tundra [18], sifting, 27.VII.2016; 8 ○ $\sigma^{7}, 36$ 우, dump sites, destroyed constructions, under rubbish items, etc. in village [20], hand collecting, 29.VII. \& 1.VIII.2016.

REMARKS. This species is new to the fauna of the Yugorsky Peninsula.

RANGE. Holarctic polyzonal.

\section{Bathyphantes humilis (L. Koch, 1879)}

MATERIAL. Zonal. 1 , undershrub-sedge-lichen-moss tundra with spots of bare ground [1], pitfall trapping, 30.VII.4.VIII.2016; 2 우, undershrub-sedge-lichen-moss tundra [3], pitfall trapping, 27.VII.-4.VIII.2016.

Intra. Monadnocks: 4 우, [5], hand collecting and sifting, 26.VII.2016. Zoogenic: 1 , grass community near polar fox burrows [6], pitfall trapping, 24-27.VII.2016. Slopes: 1 , dry DryasAstragal associations on slope in tundra [8], pitfall trapping, 14.VIII.2016. Wetlands: $1 \bigcirc^{\top} \sigma^{\top}, 2$ 우, wet willow-grassy flow bed on slope to sea shore [11], sifting and pitfall trapping, 24.VII.5.VIII.2016; 1 ○', 4 우, same locality, sifting, 4.VIII.2016; 2 ○ $\sigma^{\top}$, Equisetum-sedge swamp [12], sweeping and sifting, 28.VII.2016; $1 \sigma^{7}$, wet sedge tundra on gentle slope [13], sifting, 30.VII.2016. Amderma River Valley: $1 \sigma^{7}, 3$ 우, rocky slopes and rock outcrops in dry river bed [14], sifting and hand collecting, 31.VII., 3 \& 5.VIII.2016; 2 कo, willow shrubs in flood-plain [15], sifting and pitfall trapping, 31.VII.-5.VIII.2016.

Anthro. $1 \sigma^{7,} 3$ ㅇ, Amderma River mouth near village [17], flood-plain, under rubbish items, 30.VII.2016; 2 우, dwarf willowEquisetum tundra [18], sifting, 30.VII.2016; 8 ○', 61 우, dump sites, destroyed constructions, under rubbish items, etc. in village [20], hand collecting, 29.VII. \& 1.VIII.2016.

REMARKS. This species is new to the fauna of the Yugorsky Peninsula.

RANGE. Siberian hypoarcto-montane.

\section{Bathyphantes reprobus (Kulczyński, 1916)}

MATERIAL. Intra. Amderma River Valley: $1+$, rocky slopes and rock outcrops in dry river bed [14], sifting and hand collecting, 31.VII., 3 \& 5.VIII.2016.

REMARKS. This species is new to the fauna of the Yugorsky Peninsula.

RANGE. Fennoscandian-Siberian-Nearctic hypoarctomontane.

Bathyphantes setiger F.O. Pickard-Cambridge, 1894

MATERIAL. Intra. Wetlands: $1+$, wet willow-grassy flow bed on slope to sea shore [11], sifting, 4.VIII.2016.

REMARKS. This species is new to the fauna of the Yugorsky Peninsula.

RANGE. Palaearctic boreo-montane.

Diplocephalus barbiger (Roewer, 1955)

1988a Diplocephalus barbatus. — Eskov: 16.

MATERIAL. Intra. Slopes: 1 , dry dwarf willows-DryasAstragal tundra on slopes to sea [7], sifting, 28.VII.2016; $2 \sigma^{7} \sigma^{7}$, same locality, pitfall trapping, 25-28.VII.2016; $2 \sigma^{\top} \sigma^{\top}, 5$ 우, same locality, sifting and pitfall trapping, 24.VII.-5.VIII.2016. Wetlands: $3 \bigcirc^{\top} \sigma^{7}, 4 \bigcirc 0$, wet grassy flow bed in tundra [10], pitfall trapping, sifting and pitfall trapping, 30.VII.-4.VIII.2016; $1 \overbrace{}^{\top}$, wet willowgrassy flow bed on slope to sea shore [11], pitfall trapping, 2528.VII.2016; $2 \sigma^{\top} \sigma^{7}, 11$ +क, same locality, sifting, 4.VIII.2016; 2 $\sigma^{\top} \sigma^{\top}$, same locality, sifting and pitfall trapping, 24.VII.-5.VIII.2016; $1 \mathrm{\sigma}^{\top}$, Equisetum-sedge swamp [12], sifting, 28.VII.2016.

Anthro. 1 , , dwarf willow-Equisetum tundra [18], pitfall trapping, 24-27.VII.2016; $3 \sigma^{7} \sigma^{7}$, dump sites, destroyed constructions, under rubbish items, etc. in village [20], hand collecting, 29.VII. \& 1.VIII.2016.

REMARKS. In the Yugorsky Peninsula, this species has been recorded from Cape Belyi Nos [Eskov, 1988a, as D. barbatus].

RANGE. Siberian-Nearctic arctic.

Erigone arctica palaearctica Braendegaard, 1934

1985 Erigone arctica palaearctica. - Eskov: 123.

MATERIAL. Intra. Wetlands: $4 \sigma^{7} \sigma^{7}, 2$ 우, wet grassy flow bed in tundra [10], pitfall trapping, sifting and pitfall trapping, 30.VII.-4.VIII.2016. Amderma River Valley: $1 \sigma^{7}, 1$, rocky slopes and rock outcrops in dry river bed [14], sifting and hand collecting, 31.VII., 3 \& 5.VIII.2016.

Azon. 1 , ground bluffs above sea beach [16], hand collecting, 28.VIII.2016.

Anthro. $23 \sigma^{\top} \sigma^{\top}, 7$ 7 , Amderma River mouth near village [17], flood-plain, under rubbish items, 30.VII.2016; $3 \sigma^{7} \sigma^{7}, 1$, dwarf willow-Equisetum tundra [18], pitfall trapping, 2430.VII.2016; 4 OO, same locality, sifting, 27.VII.2016; $5 \sigma^{\top}, 3$ of, grass associations in village [19], pitfall trapping, 26.VII.5.VIII.2016; $41 \bigcirc^{7} \sigma^{7}, 40$ 우, dump sites, destroyed constructions, under rubbish items, etc. in village [20], hand collecting, 29.VII. \& 1.VIII. 2016

REMARKS. In the Yugorsky Peninsula, this species has been recorded from Cape Belyi Nos and Yugorsky Shar Polar Station [Eskov, 1985].

RANGE. East European arctic. 


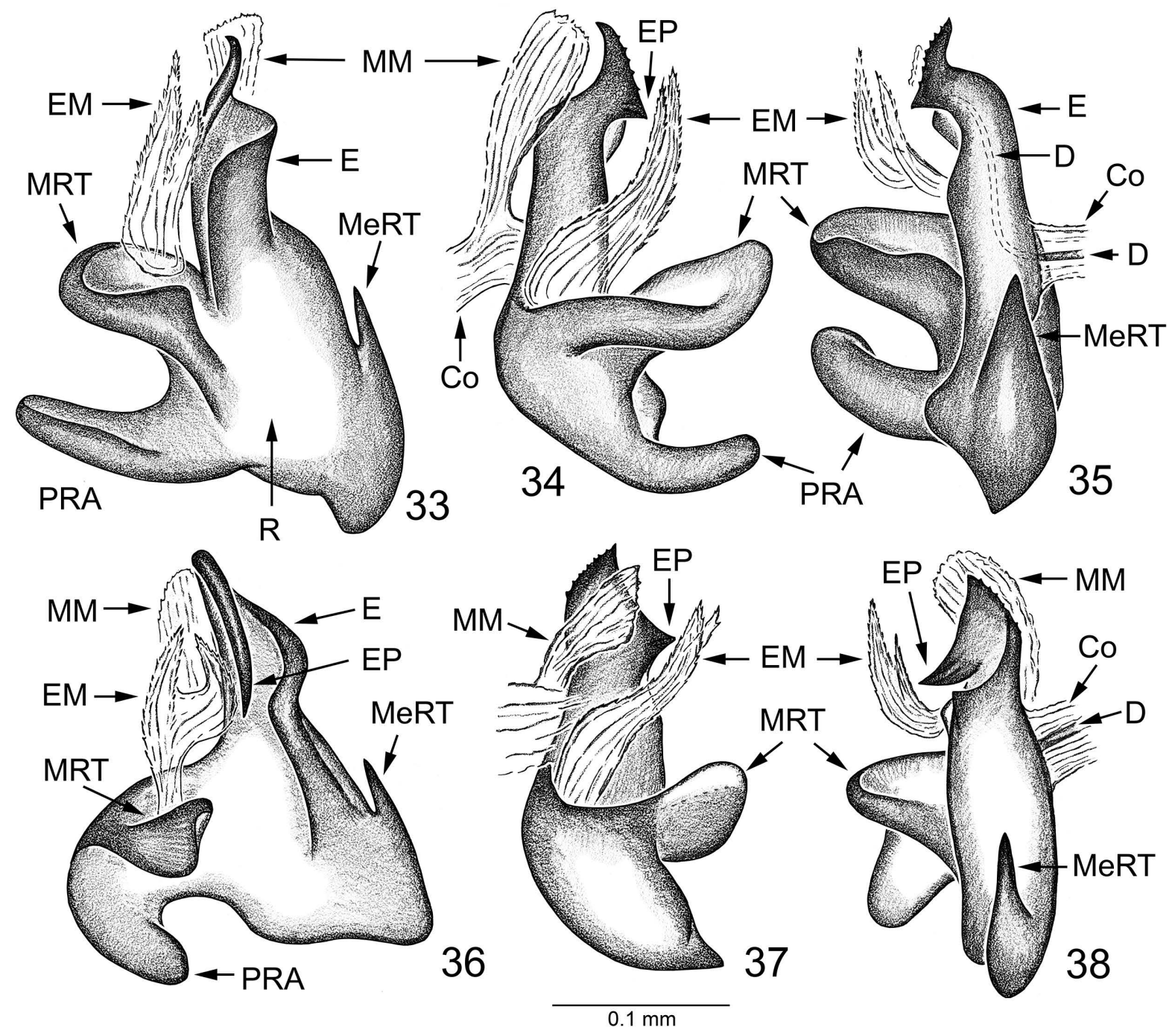

Figs 33-38. Embolic division of Erigone longipalpis (Sundevall, 1830) (33-35) and E. tirolensis L. Koch, 1872 (36-38), specimens from Amderma.

Рис. 33-38. Эмболюсный отдел Erigone longipalpis (Sundevall, 1830) (33-35) и E. tirolensis L. Koch, 1872 (36-38), экземпляры из Амдермы.

Erigone arcticola Chamberlin et Ivie, 1947

MATERIAL. Intra. Amderma River Valley: 1 , willow shrubs in flood-plain [15], sifting and pitfall trapping, 31.VII.-5.VIII.2016.

Azon. $3 O^{7} 0^{7}, 9$ 우, sea beach, under stones [16], hand collecting, 25.VIII.2016.

REMARKS. This species is new to the fauna of the Yugorsky Peninsula.

RANGE. Siberian-West Nearctic arcto-alpine.

Erigone longipalpis (Sundevall, 1830)

Figs 33-35.

MATERIAL. Intra. Amderma River Valley: 19 ㅇ, rocky slopes and rock outcrops in dry river bed [14], sifting and hand collecting, 31.VII., 3 \& 5.VIII. 2016.

Anthro. 20 우, Amderma River mouth near village [17], floodplain, under rubbish items, 30.VII.2016; $5 \sigma^{7} \sigma^{7}, 21$ क 9 , dump sites, destroyed constructions, under rubbish items, etc. in village [20], hand collecting, 29.VII. \& 1.VIII.2016.
REMARKS. This species is new to the fauna of the Yugorsky Peninsula.

RANGE. Palaearctic hypoarcto-boreo-montane.

Erigone psychrophila Thorell, 1872

1985 Erigone psychrophila. - Eskov: 124.

MATERIAL. Intra. Zoogenic. 1 , grass community near polar fox burrows [6], pitfall trapping, 24-27.VII.2016. Amderma River Valley: 1 , , swampy gully [15], mosses, sifting, 31.VII.2016.

REMARKS. In the Yugorsky Peninsula, this species has been recorded from Cape Belyi Nos and Yugorsky Shar Polar Station [Eskov, 1985].

RANGE. Holarctic arctic.

Erigone tirolensis L. Koch, 1872 Figs 36-38.

MATERIAL. Intra. Amderma River Valley: $28 \sigma^{7} \sigma^{7}$, rocky slopes and rock outcrops in dry river bed [14], sifting and hand collecting, 31.VII., 3 \& 5.VIII.2016. 
REMARKS. This species is new to the fauna of the Yugorsky Peninsula.

RANGE. Holarctic arcto-alpine.

\section{Gibothorax tchernovi Eskov, 1989}

MATERIAL. Intra. Wetlands: $7 \odot^{\top} \odot^{\top}, 9$ 9 wet willow-grassy flow bed on slope to sea shore [11], sifting and pitfall trapping, 24.VII.-5.VIII.2016; $1 \sigma^{7}, 4$ 우, Equisetum-sedge swamp [12], sifting, 28.VII.2016; $15 \mathrm{O}^{3} \mathrm{O}^{7}, 13$ 우, wet sedge tundra on gentle slope [13], sifting, 30.VII.2016.

REMARKS. This species is new to the fauna of the Yugorsky Peninsula.

RANGE. Siberian arctic.

\section{Halorates holmgreni (Thorell, 1871)}

1985 Collinsia holmgreni. - Eskov: 123.

MATERIAL. Zonal. 1 q, undershrub-sedge-lichen-moss tundra [3], sifting, 27.VII.2016.

Intra. Monadnocks: $5 \sigma^{7} \sigma^{7}, 4$ 우, [5], hand collecting and sifting, 26.VII.2016. Zoogenic: $1 \sigma^{7}, 4$ $\phi+$, grass community near polar fox burrows [6], pitfall trapping, 24-27.VII.2016. Wetlands: $1 \sigma^{7}, 1$, wet willow-grassy flow bed on slope to sea shore [11], pitfall trapping, 25-28.VII.2016; $1 \sigma^{\top}, 3$ + 9 , 3 juv., same locality, sifting, 28.VII.2016; $7 \Im^{7} \circlearrowleft^{7}, 4$ 우, same locality, sifting and pitfall trapping, 24.VII.-5.VIII.2016. Amderma River Valley: $90 \overbrace{}^{7} \sigma^{7} \&$ + , rocky slopes and rock outcrops in dry river bed [14], sifting and hand collecting, 31.VII., $3 \&$ 5.VIII.2016; 3 우, willow shrubs in flood-plain [15], sifting, 3.VIII.2016.

Azon. $5 \bigcirc^{7} \sigma^{7}$, sea beach, under stones [16], hand collecting, 25.VIII. 2016

Anthro. 1 , Amderma River mouth near village [17], floodplain, under rubbish items, 30.VII.2016; $25 \sigma^{\top} \sigma^{\top}, 23$ dwarf willowEquisetum tundra [18], pitfall trapping, 24-27.VII.2016; 50 ○ $\sigma^{7}$ $\&$ 우, same locality, sifting, 27.VII.2016; $5 \sigma^{7} \sigma^{7}$, grass associations in village [19], pitfall trapping, 26.VII.-5.VIII.2016; $9 \sigma^{7} \sigma^{7}$, 29 우, dump sites, destroyed constructions, under rubbish items, etc. in village [20], hand collecting, 29.VII. \& 1.VIII.2016.

REMARKS. In the Yugorsky Peninsula, this species has been recorded from Yugorsky Shar Polar Station [Eskov, 1985, as Collinsia h.].

RANGE. Holarctic arcto-montane.

\section{Halorates spetsbergensis (Thorell, 1872)}

1985 Collinsia spetsbergensis. - Eskov: 123.

MATERIAL. Intra. Wetlands: $7 \sigma^{7} \sigma^{7}, 8$ 우, wet grassy flow bed in tundra [10], pitfall trapping, 24-27.VII.2016; 12 O $^{\top} \sigma^{\top}, 9$ 9 , same locality, sifting and pitfall trapping, 30.VII.-4.VIII.2016.

REMARKS. In the Yugorsky Peninsula, this species has been recorded from Cape Belyi Nos [Eskov, 1985, as Collinsia s.].

RANGE. Holarctic arctic.

\section{Hilaira glacialis (Thorell, 1871)}

1985 Hilaira glacialis. - Eskov: 124.

MATERIAL. Zonal. $1 \sigma^{7}$, undershrub-sedge-lichen-moss tundra with spots of bare ground [1], pitfall trapping, 24-27.VII.2016; $5 \sigma^{\top} \sigma^{\top}, 10$ 우, same locality, sifting, 27.VII.2016; 1 \%, same locality, pitfall trapping, 24.VII.-5.VIII.2016; 1 \%, same locality, pitfall trapping, 24.VII.-5.VIII.2016; 11 कo, 1 juv., same locality, pitfall trapping, 30.VII.-4.VIII.2016; 1 +, same locality, pitfall trapping, 30.VII.-4.VIII.2016; 1 \%, undershrub-lichen-moss tundra [2], pitfall trapping, 24-27.VII.2016; $8 \sigma^{\top} \sigma^{7}, 17$ 우, same locality, sifting and pitfall trapping, 1-4.VIII.2016; 1 ㅇ, same locality, pitfall trapping, 27.VII.-1.VIII.2016; 1 \%, undershrub-sedge-lichen-moss tundra [3], pitfall trapping, 24-27.VII.2016; 4 ठ $^{7}, 2$
오, same locality, sifting, 27.VII.2016; $5 \sigma^{7} \sigma^{\top}, 1$, same locality, sifting, 27.VII.-4.VIII.2016; $3 \sigma^{\top} \sigma^{\top}, 4$ 우, dwarf willow-lichenmoss tundra [4], sifting, 2.VIII.2016.

Intra. Slopes: $1 \sigma^{7}$, dry dwarf willows-Dryas-Astragal tundra on slopes to sea [7], sifting, 28.VII.2016; $1 \sigma^{7}, 1$, same locality, sifting and pitfall trapping, 24.VII.-5.VIII.2016; $1 \sigma^{7}, 1$, same locality, sifting, 28.VII.2016; 1 9, same locality, pitfall trapping, 25-28.VII.2016; 1 ㅇ, same locality, pitfall trapping, 28.VII.5.VIII.2016; 1 フ , 3 Oᄋ, dry Dryas-Astragal associations on slope in tundra [8], sifting and pitfall trapping, 24.VII.-5.VIII.2016.

REMARKS. In the Yugorsky Peninsula, this species has been recorded from Cape Belyi Nos [Eskov, 1985].

RANGE. Siberian arcto-alpine.

\section{Hilaira incondita (L. Koch, 1879)}

1985 Hilaira incondita. - Eskov: 123.

MATERIAL. Zonal. 1 , undershrub-sedge-lichen-moss tundra [3], sifting, 27.VII.2016.

Intra. Wetlands: $1 \sigma^{7}$, Equisetum-sedge swamp [12], sifting, 28.VII.2016; 2 Oᄋ, wet sedge tundra on gentle slope [13], sifting, 30.VII.2016. Amderma River Valley: $2 \sigma^{7} \sigma^{7}$, willow shrubs in flood-plain [15], sifting, 3.VIII.2016.

Anthro. $7 \sigma^{7} \sigma^{7}, 15$ 오, Amderma River mouth near village [17], flood-plain, under rubbish items, 30.VII.2016; 1 엉 dwarf willow-Equisetum tundra [18], sifting, 27.VII.2016; 1 으, grass associations in village [19], sifting, 29.VII.2016; $11 \sigma^{\top} \sigma^{\top}, 14$ 우, dump sites, destroyed constructions, under rubbish items, etc. in village [20], hand collecting, 29.VII. \& 1.VIII.2016.

REMARKS. In the Yugorsky Peninsula, this species has been recorded from Cape Belyi Nos and Yugorsky Shar Polar Station [Eskov, 1985].

RANGE. Siberian-Nearctic arctic.

\section{Hilaira nivalis Holm, 1937}

1985 Hilaira nivalis. - Eskov: 123.

1987 H. nivalis. — Eskov: 1030.

MATERIAL. Zonal. 1 , undershrub-sedge-lichen-moss tundra [3], pitfall trapping, 27.VII.-4.VIII.2016; 1 q, undershrublichen-moss tundra [2], pitfall trapping, 24-27.VII.2016; 2 우, dwarf willow-lichen-moss tundra [4], sifting, 2.VIII.2016.

Intra. Slopes: $3 \sigma^{\top} \sigma^{\top}, 4 \bigcirc \circ$, dry dwarf willows-Dryas-Astragal tundra on slopes to sea [7], pitfall trapping, 25-28.VII.2016; 3 $\sigma^{\top} \sigma^{\top}, 2$ 우, same locality, sifting and pitfall trapping, 24.VII.5.VIII.2016; $1 \sigma^{7}$, same locality, sifting, 28.VII.2016. Wetlands: 3 $\sigma^{\top} \sigma^{\top}, 1$, wet willow-grassy flow bed on bank of brook [9], sifting and pitfall trapping, 24.VII.-5.VIII.2016; 1 ; , wet willow-grassy flow bed on slope to sea shore [11], pitfall trapping, 25-28.VII.2016; $2 \sigma^{7} \sigma^{7}, 1$, same locality, sifting and pitfall trapping, 24.VII.5.VIII.2016; 1 ऽ, Equisetum-sedge swamp [12], sifting, 28.VII.2016. Amderma River Valley: 1 q, rocky slopes and rock outcrops in dry river bed [14], sifting and hand collecting, 31.VII., 3 \& 5.VIII.2016; 3 of , willow shrubs in flood-plain [15], sifting and pitfall trapping, 31.VII.-5.VIII.2016.

Azon. $1 \sigma^{7}, 1$, sea beach, under stones [16], hand collecting, 25.VIII.2016.

Anthro. $1+$, dwarf willow-Equisetum tundra [18], pitfall trapping, 27-30.VII.2016; 2 of, same locality, sifting, 27.VII.2016.

REMARKS. In the Yugorsky Peninsula, this species has been recorded from Cape Belyi Nos [Eskov, 1985, 1987].

RANGE. West-Siberian arctic.

\section{Hilaira proletaria (L. Koch, 1879)}

1985 Hilaira proletaria. - Eskov: 123.

MATERIAL. Intra. Wetlands: $1 \sigma^{r}, 2$ ㅇ, Equisetum-sedge swamp [12], sifting, 28.VII.2016

REMARKS. In the Yugorsky Peninsula, this species has been recorded from Cape Belyi Nos and Yugorsky Shar Polar Station [Eskov, 1985]. 
RANGE. Siberian-West Nearctic arctic.

\section{Hybauchenidium aquilonare (L. Koch, 1879)}

MATERIAL. Zonal. 1 , undershrub-sedge-lichen-moss tundra with spots of bare ground [1], sifting, 27.VII.2016; 1 \%, undershrub-lichen-moss tundra [2], pitfall trapping, 24-27.VII.2016; 1 $\sigma^{7}, 1+$, same locality, sifting and pitfall trapping, 1-4.VIII.2016; 1 $\sigma^{7}, 2$ 우, undershrub-sedge-lichen-moss tundra [3], pitfall trapping, 24-27.VII.2016; 1 ऽ', same locality, sifting, 27.VII.2016; 1 $\sigma^{7}, 3$ 우, same locality, sifting, 27.VII.-4.VIII.2016.

Intra. Slopes: $1 \sigma^{7}, 2$ of, dry dwarf willows-Dryas-Astragal tundra on slopes to sea [7], sifting, 28.VII.2016; $1 \sigma^{7}$, same locality, pitfall trapping, 25-28.VII.2016; $11 \sigma^{7} \sigma^{7}, 6$ 우, same locality, sifting and pitfall trapping, 24.VII.-5.VIII.2016; $1 \sigma^{7}$, same locality, sifting, 28.VII.2016; $5 \sigma^{\top} \sigma^{\top}$, same locality, pitfall trapping, 28.VII.-5.VIII.2016. $1 \overbrace{}^{7}, 1$ subad., dry Dryas-Astragal associations on slope in tundra [8], pitfall trapping, 1-4.VIII.2016. Wetlands: 4 $+{ }_{+}$, wet willow-grassy flow bed on bank of brook [11], sifting and pitfall trapping, 24.VII.-5.VIII.2016.

Anthro. $6 \sigma^{\top} \sigma^{\top}, 9$ O+, dwarf willow-Equisetum tundra [18], pitfall trapping, 27-30.VII.2016.

REMARKS. This species is new to the fauna of the Yugorsky Peninsula.

RANGE. Siberian-West Nearctic arctic.

\section{Improphantes complicatus (Emerton, 1882)}

MATERIAL. Intra. Amderma River Valley: $15 \sigma^{\top} \sigma^{7}, 23$ 우, rocky slopes and rock outcrops in dry river bed [14], sifting and hand collecting, 31.VII., 3 \& 5.VIII.2016.

Anthro. $17 \sigma^{7} \sigma^{\top}, 15$ 우, Amderma River mouth near village [16], flood-plain, under rubbish items, 30.VII.2016.

REMARKS. This species is new to the fauna of the Yugorsky Peninsula.

RANGE. Holarctic hypoarcto-montane.

\section{Islandiana cristata Eskov, 1987}

MATERIAL. Intra. Amderma River Valley: $1 \stackrel{\circ}{\text {, rocky slopes }}$ and rock outcrops in dry river bed [14], sifting and hand collecting, 31.VII., 3 \& 5.VIII. 2016.

REMARKS. This species is new to the fauna of Europe. Amderma is westernmost locality of the known species distribution.

RANGE. Siberian-West Nearctic hypoarctic.

\section{Leptorhoptrum robustum (Westring, 1851)}

MATERIAL. Intra. Wetlands: $2 \sigma^{7} \sigma^{7}$, wet willow-grassy flow bed on slope to sea shore [11], sifting and pitfall trapping, 24.VII.5.VIII.2016. Amderma River Valley: 1 , rocky slopes and rock outcrops in dry river bed [14], sifting and hand collecting, 31.VII., 3 \& 5.VIII. 2016.

Anthro. $1 \sigma^{7}$, Amderma River mouth near village [17], floodplain, under rubbish items, 30.VII.2016; 2 o+ , dump sites, destroyed constructions, under rubbish items, etc. in village [20], hand collecting, 29.VII. \& 1.VIII.2016.

REMARKS. This species is new to the fauna of the Yugorsky Peninsula.

RANGE. Palaearctic-West Nearctic boreo-nemoral. 1875)

Macrargus multesimus (O. Pickard-Cambridge,

MATERIAL. Intra. Amderma River Valley: $7 \sigma^{7} \sigma^{\top}, 15$ \% , willow shrubs in flood-plain [15], sifting and pitfall trapping, 31.VII.-5.VIII.2016.
REMARKS. This species is new to the fauna of the Yugorsky Peninsula.

RANGE. Holarctic boreo-montane.

\section{Masikia indistincta (Kulczyński, 1908)}

1985 "Macrargus" indistinctus. - Eskov: 123.

MATERIAL. Intra. Wetlands: $1 \sigma^{7}, 2$ 우, wet willow-grassy flow bed on slope to sea shore [7], sifting and pitfall trapping, 24.VII.-5.VIII.2016; 1 フ', Equisetum-sedge swamp [12], sifting, 29.VII.2016; 1 , wet sedge tundra on gentle slope [13], sifting, 30.VII.2016.

Anthro. $3 \bigcirc^{\top} \bigcirc^{7}, 7$ 우, dump sites, destroyed constructions, under rubbish items, etc. in village [20], hand collecting, 29.VII. \& 1.VIII.2016.

REMARKS. In the Yugorsky Peninsula, this species has been recorded from Yugorsky Shar Polar Station [Eskov, 1985, as "Macrargus" indistinctus].

RANGE. Siberian-Nearctic arctic.

Mecynargus paetulus (O. Pickard-Cambridge, 1875)

MATERIAL. Anthro. $2 \sigma^{\top} \sigma^{\top}, 1$, Amderma River mouth near village [17], flood-plain, under rubbish items, 30.VII.2016; 1 웅 grass associations in village [19], sweeping, 30.VII.2016; $15 \sigma^{7} \sigma^{7}$, 14 ㅇ, dump sites, destroyed constructions, under rubbish items, etc. in village [20], hand collecting, 29.VII. \& 1.VIII.2016.

REMARKS. This species is new to the fauna of the Yugorsky Peninsula.

RANGE. Holarctic hypoarcto-boreo-montane.

Mecynargus tundricola Eskov, 1988

MATERIAL. Zonal. $3 \sigma^{7} \sigma^{r}, 3$ 우, undershrub-sedge-lichenmoss tundra [3], sifting, 27.VII.2016; $14 \bigcirc^{7} \sigma^{7}, 15$ 9 , dwarf willow-lichen-moss tundra [4], sifting, 2.VIII.2016.

REMARKS. This species is new to the fauna of the Yugorsky Peninsula.

RANGE. Siberian arctic.

Mughiphantes sobrius (Thorell, 1872)

MATERIAL. Intra. Monadnocks: $1 \sigma^{\top}, 3$ o , [5], hand collecting and sifting, 26.VII.2016. Amderma River Valley: $2 \sigma^{\top} \sigma^{\top}, 2$ 우, rocky slopes and rock outcrops in dry river bed [14], sifting and hand collecting, 31.VII., 3 \& 5.VIII.2016; 1 ㅇ, willow shrubs in flood-plain [15], sifting and pitfall trapping, 31.VII.-5.VIII.2016.

Anthro. $1 \sigma^{7}$ subad., grass associations in village [19], sweeping, 5.VIII.2016.

REMARKS. This species is new to the fauna of the Yugorsky Peninsula.

RANGE. Palaearctic arctic.

Oreoneta leviceps (L. Koch, 1879)

MATERIAL. Intra. Monadnocks: $7 \sigma^{7} \sigma^{7}, 15$ OQ , [5], hand collecting and sifting, 26.VII.2016. Slopes: $1 \sigma^{\top}$, dry dwarf willows-Dryas-Astragal tundra on slopes to sea [7], sifting and pitfall trapping, 24.VII.-5.VIII.2016. Amderma River Valley: $3 \sigma^{7} \sigma^{\top}, 22$ $\$ O$, rocky slopes and rock outcrops in dry river bed [14], sifting

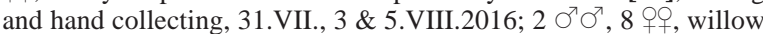
shrubs in flood-plain [15], sifting and pitfall trapping, 31.VII.5.VIII.2016.

Azon. $3 \bigcirc^{7} \sigma^{7}, 11$ 옹, sea beach, under stones [16], hand collecting, 25.VIII.2016.

Anthro. $6 \sigma^{\top} \sigma^{\top}, 7$, 7 , dump sites, destroyed constructions, under rubbish items, etc. in village [20], hand collecting, 29.VII. \& 1.VIII.2016. 
REMARKS. This species is new to the fauna of the Yugorsky Peninsula.

RANGE. Siberian Nearctic arctic.

\section{Oreoneta uralensis Saaristo et Marusik, 2004}

MATERIAL. Anthro. $1 \bigcirc^{\top}, 2$ 우, Amderma River mouth near village [17], flood-plain, under rubbish items, 30.VII.2016.

REMARKS. This species is new to the fauna of the Yugorsky Peninsula.

RANGE. East European-West Siberian hypoarctic.

\section{Pelecopsis parallela (Wider, 1834)}

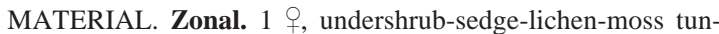
dra with spots of bare ground [1], pitfall trapping, 24-27.VII.2016; 1 , undershrub-lichen-moss tundra [2], pitfall trapping, 2427.VII.2016.

Intra. Wetlands: $1+$, wet willow-grassy flow bed on slope to sea shore [11], sweeping, 2.VIII.2016; 1 \%, Equisetum-sedge swamp [12], sweeping, 28.VII.2016; 1 \%, same locality, sifting, 29.VII.2016; $1 \mathrm{O}^{7}$, same locality, pitfall trapping, 2-5.VIII.2016.

Anthro. 2 우, grass associations in village [19], sifting, 29.VII.2016; 2 +o, dump sites, destroyed constructions, under rubbish items, etc. in village [20], hand collecting, 29.VII. \& 1.VIII.2016.

REMARKS. This species is new to the fauna of the Yugorsky Peninsula.

RANGE. Palaearctic polyzonal.

\section{Proislandiana pallida (Kulczyński, 1908)}

MATERIAL. Intra. $3 \sigma^{7} \sigma^{7}, 1$, rock outliers in tundra [5], hand collecting and sifting, 26.VII.2016. Amderma River Valley: 1 $\mathrm{O}^{7}, 1+$, rocky slopes and rock outcrops in dry river bed [14], sifting and hand collecting, 31.VII., 3 \& 5.VIII. 2016.

REMARKS. This species is new to the fauna of Europe. Amderma is westernmost locality of the known species distribution.

RANGE. Siberian arcto-montane.

\section{Scotinotylus evansi (O. Pickard-Cambridge, 1894)}

MATERIAL. Intra. Amderma River Valley: $80^{7} 0^{7}, 32$ 우, rocky slopes and rock outcrops in dry river bed [14], sifting and hand collecting, 31.VII., 3 \& 5.VIII.2016; $3 \sigma^{7} \sigma^{7}, 3$ 우, willow shrubs in flood-plain [15], sifting and pitfall trapping, 31.VII.5.VIII.2016.

REMARKS. This species is new to the fauna of the Yugorsky Peninsula.

RANGE. European-West Siberian hypoarcto-montane.

\section{Semljicola alticola (Holm, 1950)}

MATERIAL. Zonal. $1 \sigma^{\text {, }}$, undershrub-lichen-moss tundra [2], sifting and pitfall trapping, 24-27.VII.2016; $1 \bigcirc^{7}$, same locality, sifting and pitfall trapping, 1-4.VIII.2016.

Intra. Slopes: $1{ }^{\nearrow}, 3$ 우, dry dwarf willows-Dryas-Astragal tundra on slopes to sea [7], sweeping, 2.VIII.2016. Zoogenic: 1 , grass community near polar fox burrows [6], pitfall trapping, 2427.VII.2016. Wetlands: $1 \sigma^{7}$, wet grassy flow bed in tundra [10], 24-27.VII.2016; $11 \bigcirc^{7} \sigma^{7}, 8$ 우, same locality, sifting and pitfall trapping, 30.VII.-4.VIII.2016; $2 \sigma^{7} \sigma^{7}, 9$ 우, wet willow-grassy flow bed on bank of brook [9], sifting and pitfall trapping, 24.VII.-

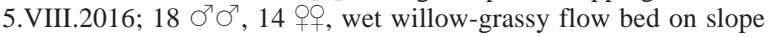
to sea shore [11], sifting and pitfall trapping, 24.VII--5.VIII.2016; $1 O^{\top}, 1$ ㅇ, same locality, sifting, 28.VII.2016; $4 \sigma^{\top} O^{\top}$, same locality, sweeping, 2.VIII.2016; $9 \sigma^{\top} \sigma^{\prime}, 12$ 우, Equisetum-sedge swamp
[12], sweeping, 28.VII.2016; $3 \sigma^{\top} \sigma^{\top}, 4$ 우, same locality, sifting, 28.VII.2016; $1 \mathrm{O}^{\mathrm{T}}, 2$ 우, same locality, sifting, 29.VII.2016; $1 \mathrm{O}^{\mathrm{T}}$, same locality, pitfall trapping, 2-5.VIII.2016; $33 \mathrm{O}^{7} \sigma^{7}, 25$ of , wet sedge tundra on gentle slope [13], sifting, 30.VII.2016. Amderma River Valley: $5 \bigcirc^{7} \sigma^{7}, 6$ ㅇ, rocky slopes and rock outcrops in dry river bed [14], sifting and hand collecting, 31.VII., 3 \& 5.VIII.2016; $37 \sigma^{7} \sigma^{7}, 43$ ofo, willow shrubs in flood-plain [15], sifting and pitfall trapping, 31.VII--5.VIII.2016; 1 , swampy gully [15], mosses, sifting, 31.VII.2016.

Azon. $1 \sigma^{7}$, sea beach, under stones [16], hand collecting, 25.VIII.2016; 1 , ground bluffs above sea beach [16], pitfall trapping, 25-28.VIII.2016.

Anthro. $11{\sigma^{7}}^{7} \sigma^{7}, 18$ 웅, Amderma River mouth near village [17], flood-plain, under rubbish items, 30.VII.2016; $1 \sigma^{7}, 19$, dwarf willow-Equisetum tundra [18], pitfall trapping, 2427.VII.2016; 2 \%, grass associations in village [19], sweeping, 29 \& 30.VII.2016; $29 \bigcirc^{7} \sigma^{7}, 49$ 우, dump sites, destroyed constructions, under rubbish items, etc. in village [20], hand collecting, 29.VII. \& 1.VIII.2016

REMARKS. This species is new to the fauna of the Yugorsky Peninsula.

RANGE. Fennoscandian-Siberian arctic.

\section{Semljicola arcticus (Eskov, 1989)}

1985 Latithorax sp. - Eskov: 124.

1989 Latithorax arcticus. - Eskov: 101.

MATERIAL. Zonal. 1 , undershrub-sedge-lichen-moss tundra with spots of bare ground [1], pitfall trapping, 24-27.VII.2016; $2 \bigcirc^{\top} \sigma^{\top}, 5$ 우, same locality, sifting, 27.VII.2016; 1 , same locality, pitfall trapping, 30.VII.-4.VIII.2016; $5 \sigma^{\top} \sigma^{\top}, 9$ 우, undershrublichen-moss tundra [2], sifting and pitfall trapping, 1-4.VIII.2016; $1 \mathrm{O}^{7}, 1 \stackrel{\circ}{ }$, dwarf willow-lichen-moss tundra [4], sifting, 2.VIII.2016,

Intra. Monadnocks: $7 \sigma^{7} \sigma^{7}, 15$ 우, [5], hand collecting and sifting, 26.VII.2016. Slopes: $1 \sigma^{7}$, dry dwarf willows-Dryas-Astragal tundra on slopes to sea [7], sifting, 28.VII.2016.

REMARKS. In the Yugorsky Peninsula, this species has been recorded from Cape Belyi Nos [Eskov, 1985, as Latithorax sp.], and from Yugorsky Shar Polar Station [Eskov, 1989].

RANGE. Siberian arctic.

\section{Semljicola barbiger (L. Koch, 1879)}

1985 Eboria assimilis. - Eskov: 123.

2017 Semljicola barbiger. - Tanasevitch: 152.

MATERIAL. Zonal. $1 \sigma^{7}$, undershrub-sedge-lichen-moss tundra with spots of bare ground [1], pitfall trapping, 27.VII.4.VIII.2016; $4 \mathrm{O}^{\top} \sigma^{\top}, 8$ 우, same locality, sifting, 27.VII.2016; 1 , same locality, pitfall trapping, 30.VII.-4.VIII.2016; $3 \mathrm{O}^{7} \sigma^{7}, 11$ 우, undershrub-lichen-moss tundra [2], sweeping, 2.VIII.2016; $12 \mathrm{O}^{7} \mathrm{O}^{7}$, 15 우 , same locality, sifting and pitfall trapping, 1-4.VIII.2016; 5 웅, undershrub-sedge-lichen-moss tundra [3], sweeping, 30.VII.2016; 15 O $^{\top} \sigma^{\top}, 28$ 우, same locality, sifting, 27.VII.2016; $15 \odot^{7} \sigma^{7}, 12$ 우, dwarf willow-lichen-moss tundra [4], sifting, 2.VIII.2016.

Intra. Monadnocks: 4 ++ , [5], hand collecting and sifting, 26.VII.2016. Zoogenic: 1 , grass community near polar fox burrows [6], sweeping, 2.VIII.2016. Slopes: 19 , dry Dryas-Astragal associations on slope in tundra [8], sweeping, 2.VIII.2016; $1 \mathrm{O}^{\text {? }}$, same locality, pitfall trapping, 1-4.VIII.2016. Wetlands: $1 \bigcirc^{7}$, wet willow-grassy flow bed on bank of brook [9], sweeping, 2.VIII.2016; $3 O^{7} O^{7}, 7$ 우, same locality, sifting and pitfall trapping, 24.VII.5.VIII.2016. Amderma River Valley: 2 우, rocky slopes and rock outcrops in dry river bed [14], sifting and hand collecting, 31.VII., $3 \&$ 5.VIII.2016; $1 \sigma^{2}$, willow shrubs in flood-plain [15], sifting and pitfall trapping, 31.VII.-5.VIII.2016.

Anthro. 1 , dwarf willow-Equisetum tundra [18], sifting, 30.VII.2016.

REMARKS. In Yugorsky Peninsula, this species has been recorded from Cape Belyi Nos [Eskov, 1985, as Eboria assimilis Holm, 1963; Tanasevitch, 2017b]. 
RANGE. Fennoscandian-Siberian arcto-alpine.

\section{Semljicola simplex (Kulczyński, 1908)}

MATERIAL. Intra. Wetlands: 2 +, wet willow-grassy flow bed on bank of brook [9], sifting and pitfall trapping, 24.VII.5.VIII.2016; 1 9, Equisetum-sedge swamp [12], sifting, 29.VII.2016; 1 9 , same locality, sweeping, 28.VII.2016. Amderma River Valley: $1+$, rocky slopes and rock outcrops in dry river bed [14], sifting and hand collecting, 31.VII., $3 \& 5$.VIII.2016; $1 \sigma^{\text {T }, ~} 3$ 우, swampy gully [15], mosses, sifting, 31.VII.2016.

Anthro. 1 , dump sites, destroyed constructions, under rubbish items, etc. in village [20], hand collecting, 29.VII. \& 1.VIII.2016.

REMARKS. This species is new to the fauna of the Yugorsky Peninsula.

RANGE. Siberian arctic.

Silometopoides sphagnicola Eskov et Marusik, 1992

MATERIAL. Intra. Wetlands: $1 \sigma^{r}, 2$ $2 \circ$, wet willow-grassy flow bed on slope to sea shore [11], sifting, 4.VIII.2016; 1 , wet sedge tundra on gentle slope [13], sifting, 30.VII.2016. Amderma River Valley: 2 우, swampy flow bed [15], mosses, sifting, 31.VII.2016.

Anthro. 1 , grass associations in village [19], sifting, 29.VII.2016.

REMARKS. This species is new to the fauna of the Yugorsky Peninsula.

RANGE. Siberian hypoarcto-boreo-montane.

\section{Tarsiphantes latithorax Strand, 1905}

1985 Typhochraestus latithorax. — Eskov: 124.

REMARKS. This species is not presented in our material. In Yugorsky Peninsula this species has been recorded from Cape Belyi Nos [Eskov, 1985, as Typhochraestus (sic!) latithorax].

RANGE. Siberian-Nearctic arcto-alpine.

\section{Tenuiphantes nigriventris (L. Koch, 1879)}

MATERIAL. Zonal. 1 , undershrub-sedge-lichen-moss tundra [3], sweeping, 30.VII.2016.

Intra. Wetlands: $1 \sigma^{7}$, wet willow-grassy flow bed on bank of brook [9], sweeping, 2.VIII.2016.

Anthro. $2 \sigma^{7} \sigma^{7}, 2$, 20 , grass associations in village [19], sweeping, 30.VII.2016; 1 \% dump sites, destroyed constructions, under rubbish items, etc. in village [20], hand collecting, 29.VII. \& 1.VIII.2016.

REMARKS. This species has been recorded from vicinities of the south-eastern border of the Yugorsky Peninsula, from mouth of Kara River [Kulczyński, 1916]. This species is new to the fauna of the Yugorsky Peninsula.

RANGE. Palaearctic hypoarcto-boreo-montane.

\section{Tmeticus nigriceps (Kulczyński, 1916)}

MATERIAL. Intra. Wetlands: 1 , wet willow-grassy flow bed on slope to sea shore [11], sifting and pitfall trapping, 24.VII.5.VIII.2016; 1 옹 same locality, sifting, 4.VIII.2016; 1 , Equisetum-sedge swamp [12], sifting, 29.VII.2016; 1 \%', same locality, pitfall trapping, 2-5.VIII.2016; 1 , wet sedge tundra on gentle slope [13], sifting, 30.VII.2016. Amderma River Valley: 1 , rocky slopes and rock outcrops in dry river bed [14], sifting and hand collecting, 31.VII., 3 \& 5.VIII.2016; $1 \sigma^{7}, 2$ +o, swampy gully [15], mosses, sifting, 31.VII.2016.
Azon. $6 \sigma^{7} \sigma^{7}, 12$ ๆ , sea beach, under stones [16], hand collecting, 25.VIII.2016.

Anthro. $7 \sigma^{\top} \sigma^{\top}, 21$ ++ , Amderma River mouth near village [17], flood-plain, under rubbish items, 30.VII.2016; 7 O $^{7} \sigma^{7}, 3$ 우, grass associations in village [19], pitfall trapping, 26.VII.5.VIII.2016; $5 \sigma^{\top} \sigma^{\top}, 42$ क्+, dump sites, destroyed constructions, under rubbish items, etc. in village [20], hand collecting, 29.VII. \& 1.VIII.2016.

REMARKS. This species is new to the fauna of the Yugorsky Peninsula.

RANGE. Siberian hypoarcto-montane.

\section{Tubercithorax subarcticus (Tanasevitch, 1984)}

1985 Rhaebothorax subarcticus. - Eskov: 124.

MATERIAL. Zonal. $2 \sigma^{\top} \sigma^{\top}, 11$ 우, undershrub-sedge-lichenmoss tundra with spots of bare ground [1], sifting, 27.VII.2016.

Intra. Monadnocks: $1 \Im^{7},[5]$, hand collecting and sifting, 26.VII.2016. Slopes: $7 \sigma^{\top} \sigma^{7}, 23$ 우, dry Dryas-Astragal associations on slope in tundra [8], sifting and pitfall trapping, 24.VII.5.VIII.2016. Wetlands: $1 \sigma^{7}$, wet willow-grassy flow bed on bank of brook [9], sifting and pitfall trapping, 24.VII.-5.VIII.2016. Amderma River Valley: 1 , rocky slopes and rock outcrops in dry river bed [14], sifting and hand collecting, 31.VII., $3 \&$ 5.VIII.2016.

REMARKS. In the Yugorsky Peninsula, this species has been recorded from Cape Belyi Nos [Eskov, 1985, as Rhaebothorax s.].

RANGE. Siberian hypoarctic.

\section{Walckenaeria clavicornis (Emerton, 1882)}

1985 Cornicularia clavicornis. - Eskov: 125.

MATERIAL. Zonal. $1 \sigma^{7}, 1$, undershrub-sedge-lichen-moss tundra [3], sifting, 27.VII.2016.

Intra. Slopes: 2 + ${ }^{\circ}$, dry dwarf willows-Dryas-Astragal tundra on slopes to sea [7], sifting and pitfall trapping, 24.VII.-5.VIII.2016. Amderma River Valley: 1 o, willow shrubs in flood-plain [15] sifting and pitfall trapping, 31.VII.-5.VIII.2016.

Azon. 1 , sea beach, under stones [7], hand collecting, 25.VIII.2016; 1 , ground bluffs above sea beach [7], hand collecting, 28.VIII.2016.

REMARKS. In Yugorsky Peninsula, this species has been recorded from Cape Belyi Nos [Eskov, 1985, as Cornicularia $c$.]

RANGE. Holarctic arcto-montane.

Fam. Lycosidae

Alopecosa hirtipes (Kulczyński, 1907)

MATERIAL. Intra. Amderma River Valley: $1 q$, rocky slopes and rock outcrops in dry river bed [14], hand collecting, 31.VII.2016.

REMARKS. This species is new to the fauna of the Yugorsky Peninsula.

RANGE. Siberian-Nearctic arctic.

\section{Alopecosa mutabilis (Kulczyński, 1908)}

MATERIAL. Zonal. 2 오, 3 juv., undershrub-sedge-lichenmoss tundra with spots of bare ground [1], pitfall trapping, 2427.VII.2016; 11 कo , 4 juv., same locality, pitfall trapping, 30.VII.5.VIII.2016; 1 ㅇ, undershrub-lichen-moss tundra [2], pitfall trapping, 24-27.VII.2016; 4 कㅇ, 2 juv., same locality, sifting and pitfall trapping, 1-4.VIII.2016; 6 우, same locality, sifting and pitfall trapping, 27.VII.-1.VIII.2016; 21 우, undershrub-sedgelichen-moss tundra [3], pitfall trapping, 24.VII.-4.VIII.2016.

Intra. Zoogenic: 2 ㅇ, grass community near polar fox burrows [6], pitfall trapping, 27.VII.-1.VIII.2016. Slopes: 3 우, dry 
Dryas-Astragal associations on slope in tundra [8], pitfall trapping, 24-27.VII.2016; 8 우, same locality, pitfall trapping, 14.VIII.2016.

REMARKS. This species is new to the fauna of the Yugorsky Peninsula.

RANGE. Siberian-West Nearctic arctic.

Alopecosa solivaga (Kulczyński, 1901)

1985 Alopecosa solivaga. - Eskov: 125.

REMARKS. This species is not presented in our material. In the Yugorsky Peninsula, A. solivaga has been recorded from Yugorsky Shar Polar Station [Eskov, 1985].

RANGE. Siberian hypoarcto-montane.

Fam. Tetragnathidae

Pachygnatha clercki Sundevall, 1823

MATERIAL. Intra. Wetlands: $1 \sigma^{\prime}$, wet willow-grassy flow bed on slope to sea shore [11], pitfall trapping, 25-28.VII.2016; 2 우, same locality, sifting, 4.VIII.2016; $1 \sigma^{\gamma}$, Equisetum-sedge swamp [12], sifting, 28.VII.2016.

Azon. 1 , ground bluffs above sea beach [7], pitfall trapping, 2-5.VIII.2016.

Anthro. 1 , dwarf willow-Equisetum tundra [18], sifting, 27.VII.2016; 2 oᄋ, 1 juv., dump sites, destroyed constructions, under rubbish items, etc. in village [20], hand collecting, 29.VII. \& 1.VIII.2016.

REMARKS. This species is new to the fauna of the Yugorsky Peninsula.

RANGE. Holarctic polyzonal.

Fam. Theridiidae

Thymoites oleatus (L. Koch, 1879)

MATERIAL. Intra. Zoogenic: 1 subad., grass community near polar fox burrows [6], sweeping, 2.VIII.2016. Amderma River Valley: 1 , rocky slopes and rock outcrops in dry river bed [14], sifting and hand collecting, 31.VII., 3 \& 5.VIII.2016.

Azon. 1 o subad., ground bluffs above sea beach [16], hand collecting, 25.VIII.2016.

REMARKS. This species is new to the fauna of the Yugorsky Peninsula.

RANGE. Siberian-Nearctic hypoarcto-montane.

Fam. Thomisidae

Ozyptila arctica Kulczyński, 1908

MATERIAL. Intra. Wetlands: 1 , wet grassy flow bed in tundra [10], sweeping, 4.VIII.2016.

REMARKS. This species is new to the fauna of the Yugorsky Peninsula.

RANGE. Fennoscandian-Siberian-Nearctic hypoarctomontane.

\section{Xysticus albidus Grese, 1909}

MATERIAL. Zonal. $1 \sigma^{7}$, undershrub-sedge-lichen-moss tundra with spots of bare ground [1], pitfall trapping, 24-27.VII.2016; 1 juv., same locality, sifting, 27.VII.2016; 2 우, same locality, pitfall trapping, 24.VII.-5.VIII.2016; 1 juv., same locality, pitfall trapping, 30.VII.-4.VIII.2016; 4 o+ , undershrub-lichen-moss tundra [2], sifting and pitfall trapping, 1-4.VIII.2016; $1 \sigma^{7}, 1$ sub- ad., undershrub-sedge-lichen-moss tundra [3], pitfall trapping, 24.VII.-4.VIII.2016.

Intra. Monadnocks: $7 \sigma^{\top} \sigma^{\top}, 15$ $+{ }_{+},[5]$, hand collecting and sifting, 26.VII.2016. Zoogenic: $1 \sigma^{7}$, grass community near polar fox burrows [6], in moss, hand collecting, 24.VII.2016. Slopes: 1 $\sigma^{7}, 1 \sigma^{7}$ subad., dry Dryas-Astragal associations on slope in tundra [8], pitfall trapping, 24-27.VII.2016; 2 o+, same locality, pitfall trapping, 1-4.VIII.2016. Amderma River Valley: 1 , rocky slopes and rock outcrops in dry river bed [14], sifting and hand collecting, 31.VII., 3 \& 5.VIII.2016; 3 우, willow shrubs in flood-plain [15], sifting and pitfall trapping, 31.VII.-5.VIII.2016; $1 \sigma^{7}$ subad., same locality, sweeping, 31.VII.2016.

Anthro. $1 \sigma^{7}$ subad., Amderma River mouth near village [17] flood-plain, sweeping, 31.VII.2016.

REMARKS. This species is new to the fauna of the Yugorsky Peninsula.

RANGE. Fennoscandian-Siberian arctic.

Species from territories adjacent to the $\mathrm{Yu}-$ gorsky Peninsula

Several species of spiders from the extreme northeast of European tundra were recorded by Kulczyński [1916] who based on the results of the 1909 expedition to the Polar Urals and the Kara tundra that was funded by tea merchants, brothers N.G. and G.G. Kuznetsov. The expedition collected material in eastern Kara tundra, specifically in riverside area between the Kara River and northernmost branches of the Polar Urals. Unfortunately, the paper [op. cit.] lacks data on specific localities of collection, nor it contains information about biotopes were the spiders were collected. In total, eight spider species have been collected in the Kara River lower reaches and in Kara tundra: Alopecosa hirtipes (Kulczyński, 1907) (as Tarentula hirta Kulczyński, 1908), A. mutabilis (Kulczyński, 1908) (as Tarentula m.), A. solivaga (Kulczyński, 1901) (as Tarentula s.), Dictyna major Menge, 1869 (as D. sibiricae var. simulans Kulczyński, 1916), Erigone arctica palaearctica Braendegaard, 1934 (as E. a. sibirica Kulczyński, 1908), Pachygnatha clercki Sundevall, 1823, Pardosa tesquorum (Odenvall, 1901) and Tenuiphantes nigriventris (L. Koch, 1879) (as Lephthyphantes nigriventer, sic!). Our material from typical tundra of Amderma vicinities lacks $A$. solivaga and $P$. tesquorum: the former probably was simply not found, and $P$. tesquorum is absent in the area because the northern limit of species range lies southward of the subzone of typical tundra.

\section{Results and Discussion}

\section{Interesting records}

Perhaps, only two interesting records in studied area can be noted: Islandiana cristata and Proislandiana pallida. Both species are petrophilous; the nearest to Amderma record of the former species was in the southern Yamal [Eskov, 1987], that of the latter eastern macroslope of the Polar Urals [Tanasevitch, 1985]. Amderma is the westernmost locality of distribution of both species, and the first record in Europe. 


\section{Composition of araneofauna}

Fifty three spiders species from seven families have been collected in Amderma vicinities. Of them, 45 species ( $85 \%$ of the araneofauna) belong to Linyphiidae, the other families are represented by 1-2 species. To analyze the fauna, we used the faunistic index proposed by Medvedev [1984], which successfully used in comparison and analysis of regional faunas (see Esyunin \& Styopina [2014], Esyunin [2015], etc.). This method allows to characterize clearly (as a formula) the composition and structure of domination at the family level in one or another regional fauna. Faunistic taxonomical index (FTI) of araneofauna of the studied area of typical tundra of the Yugorsky Peninsula is described as Lin(85)-Lyc(4)-Tho(4), and the fauna can be characterized as oligotaxon linyphiid or even monotaxon (in the formula, the percentage of the family representatives is given in parentheses). Oligotaxon linyphiid faunas are characteristic feature of subarctic and, especially, arctic belt. Families Linyphiidae and Lycosidae prevail in all well studied araneofaunas of the subzone of typical tundra: FTI of araneofauna of Dolgy Island - Lin(85)-Lyc(8)-Tho(2), Tyylaakh Lin(87)-Lyc(5)-Tho(2), Tiksi - Lin(73)-Lyc (9)Tho(6)-The(6) (data from Marusik et al. [2016] and Tanasevitch \& Nekhaeva [2016]). This sequence makes clear that the local fauna of typical tundra of the Yugorsky Peninsula is distinguished by a lower percentage of wolf spiders which is rather more typical for more northern arctic-tundra faunas where Linyphiidae comprise from 89\% (northern Yamal, wolf spiders are represented by a single species) to $95-100 \%$ of the fauna (Novaya Zemlya, Vaygach, wolf spiders are absent).

\section{Zoogeographical and fraction composition of ara- neofauna}

The studied region is situated at the border between Europe and Siberia, which defines peculiarities of the zoogeographical structure of its fauna. Among 30 species of Holarctic group (with the range situating on two continents), the vast majority of species have mostly Siberian distribution (the main part of the range is in Siberia). In the Palearctic group, most species (18 of 23) have also mostly Siberian distribution; only three species (Bathyphantes setiger, Erigone longipalpis and Pelecopsis parallela) are widely distributed in the Palearctic, and only two species (Erigone arctica palaearctica and Scotinotylus evansi) occur mostly in Europe. Based on this, the fauna of typical tundra of the Yugorsky Peninsula can be characterized as Siberian, with a high share of widely distributed species and a small proportion of European and East Palearctic elements. The total share of Siberian and Siberian-Nearctic elements comprises about $52 \%$ of the fauna (27 species); their importance is especially high among spiders of arctic and hypoarctic fractions, whereas species with wider Holarctic ranges prevail in other frac- tions. In the share of Siberian and Siberian-Nearctic species, the araneofauna of Amderma is similar to the fauna of spiders of Siberian tundras (see Khruleva \& Berezin [2002]; Osipov [2003]; Tanasevitch \& Rybalov [2015]; Tanasevitch \& Nekhaeva [2016]).

More than a half of species (53\% of the fauna) belong to the arctic fraction which includes 28 species, most of them (20 species) with arctic distribution; other 8 species have arcto-montane (mainly arcto-alpine) ranges. The second most important fraction, quite expectedly, is hypoarctic one which comprises more $30 \%$ of the fauna. Species of boreal and polyzonal fractions are very poorly represented (4 and 5 species, respectively), forming together about $17 \%$ of the total list. Polyzonal elements are represented by species with rather wide occurrence in northern part of the tundra zone of Eurasia, i.e., Pelecopsis parallela, Larinioides cornutus, Dictyna major and Pachygnatha clercki [Khruleva, 1987; Tanasevitch, Koponen, 2007; Tanasevitch, Rybalov, 2015; Tanasevitch, Nekhaeva, 2016], etc. The composition of boreal species is also not accidental, all of them are actively exploring the East European tundra landscapes and were found in various areas of the Bolshezemelskaya tundra [Tanasevitch, Koponen, 2007].

At the level of species richness and in peculiarities of longitudinal and latitudinal composition, the araneofauna of Amderma vicinities is highly similar to the fauna of spatially close Dolgy Island, Barents Sea. Both of them differ from Siberian faunas by markedly higher diversity of "southern" elements (hypoarctic, boreal and polyzonal species), but only about $30 \%$ of all recorded "southern" species (11 of 39) are common for both faunas. On the contrary, there is very high similarity of the composition of spiders from the arctic fraction in faunas of Amderma and other arctic regions of Cisuralean sector: 19 species of 35 (54\%) are common with the fauna of Dolgy Island, 21 species of 32 $(66 \%)$ are common with the fauna of the Novaya Zemlya Archipelago and the Vaygach Island [Tanasevitch, 2017c]. Exactly the constancy of the set of arctic species determines the rather high similarity of faunas of Cisuralean sector between each other: Amderma Dolgy - 57\%, Amderma - northern islands (Novaya Zemlya and Vaygach) - 61\%, Dolgy and northern islands - 50\% (we used the Czekanowski-Sörensen index for qualitative data [Pesenko, 1982]).

Peculiarities of the fauna and population of spiders in different types of plant communities

\section{Zonal tundra communities (Zonal)}

Fauna of zonal tundra contains 17 species (see Table 1). Two species, Arcterigone pilifrons and Mecynargus tundricola, are specific for zonal tundra communities and have not been found outside them. In zonal aspect the fauna of plain tundra (= plakor) is mostly represented by arctic elements: the share of 
purely arctic species makes up 53\% here, and the entire arctic fraction $-76 \%$ of the fauna. Hypoarctic fraction comprises only $18 \%$, polyzonal $-6 \%$ (1 species only), species of boreal fraction are absent. Among main types of plant communities (zonal, intrazonal, azonal and anthropogenic) the share of arctic fraction is the highest in zonal tundra.

Species composition of spiders is rather stable in different areas of zonal tundra: 9 species were recorded at least in two of three biotopes studied in detail (where all the methods were used by - pitfall traps, sifting, net-sweeping); their general number varies within 10-12 species in each biotope. In the composition of dominants (species with no less than 5\% share of the specimens in the biotope population) these spider assemblages are also similar to each other. Arctic species comprise the main part of the population (89-100\%); Alopecosa mutabilis, Hilaira glacialis and Semljicola barbiger are common everywhere. In plots of neutral exposition a high abundance was recorded also for Arcterigone pilifrons and Semljicola arctica, whereas in the zonal plots on the slope of southern exposition - Hybauchenidium aquilonare and Mecynargus tundricola.

The presence of specific dominants in zonal tundra is the most specific feature of spider distribution in the studied area. Most of them highly decrease their abundance in other biotopes. It is noticeable that these groups are of clearly expressed Siberian composition - dominants are Siberian: Semljicola arcticus, Mecynargus tundricola, Hilaira glacialis; Siberian-West Nearctic: Arcterigone pilifrons, Alopecosa mutabilis and Fennoscandian-Siberian: Semljicola barbiger, Xysticus albidus. It is also interesting that all these species in well studied tundra regions of Siberia and the Far East are not restricted by zonal tundra but usually inhabit a wider range of biotopes, e.g., in northern Yamal [Tanasevitch, Rybalov, 2015], Taymyr [Khruleva, Berezin, 2002; Osipov, 2003], in lower reaches of the Lena River [Tanasevitch, Nekhaeva, 2016], Wrangel Island [Khruleva, 1987], etc.

\section{Intrazonal tundra communities (Intra)}

Fauna of spiders of intrazonal tundra communities as a whole (including the Amderma River Valley) consists of 48 species. Only 5 species of the general list of local fauna are absent here: 2 were recorded only in zonal tundra (see above) and 3 - Dictyna major, Mecynargus paetulus and Oreoneta uralensis - were found only in anthropogenic communities. The broad range of intrazonal tundra communities has defined the richness and variety of species composition. The share of purely arctic elements comprises here $38 \%$, and that of the entire arctic fraction - 54\%; the share of hypoarctic elements markedly exceeds the same index for zonal tundra $-29 \%$, the shares of boreal and polyzonal fractions are the same $-8 \%$. There is strong variability in the structure of latitudinal fractions in the fauna and population of different kinds of intrazonal communities (see below).
Monadnocks (rock outliers, rock outcrops). Thirteen species have been collected in this biotope, 8 of them ( $62 \%$ of the fauna of the biotope) belong to arctic, 3 species $(23 \%)$ - to hypoarctic, $2(15 \%)$ - to polyzonal fractions; boreal species are absent. Most part of the population ( $89 \%$ of all specimens) consists of species from arctic fraction; species from other fractions do not play a considerable role in the assemblages. Dominants of the population include Agyneta nigripes, Oreoneta leviceps, Halorates holmgreni, Xysticus albidus, one of the dominants (Semljicola arcticus) is common with zonal tundra.

Zoogenic meadow. This is the smallest of studied intrazonal plant communities, which is surrounded by zonal tundra. Only 8 species have been collected here, with prevalence of arctic fraction $-75 \%$, which is the same proportion as in zonal tundra (76\%). Most spider species collected from zoogenic meadow (except for Erigone psychrophila and Thymoites oleatus) occur also in zonal tundra. Therefore, we incline to consider the high share of species of the arctic fraction as an artifact explained by small area of this biotope and by its surroundings. Population of spiders of this area is scanty: almost all the species except for Halorates holmgreni have been found here as a single specimen.

Slopes of hills. These well drained biotopes are monotonous in composition and participation of different vegetative components. Their araneofauna includes 16 species, and in both taxonomic and chorological composition these sites are generally most similar to zonal tundra. For example, the share of purely arctic species is $50 \%$, while that of species of the entire arctic fraction is $75 \%$. Despite similarity in vegetation of slopes of different exposition, the spider assemblages somewhat varies in these habitats. Seven species were collected on a slope of southern exposition, 5 of them belong to the arctic fraction (Hilaira glacialis, Hybauchenidium aquilonare, Semljicola barbiger, Alopecosa mutabilis, Xysticus albidus, all being common with zonal tundra). The species assemblage (65\% of all collected specimens) is composed by high abundance of a single species, hypoarctic Tubercithorax subarcticus. The slopes of northern exposition and directed towards the sea yielded 11 collected species. Here, both the fauna and the population show an absolute prevalence of arctic species (82 and 96\%, respectively). Dominants of the population include Agyneta $n i$ gripes, Diplocephalus barbiger, Hilaira glacialis, Hilaira nivalis, Hybauchenidium aquilonare and Semljicola alticola, which defines similarity of spider assemblage of this biotope with both Zonal and Wetlands.

Wetlands. Wet habitats on the watershed are mainly situated on brook banks, in flowing beds and in depressions of a relief with weak drainage. The fauna of wetlands includes 26 species. The shares of purely arctic elements and the entire arctic fraction are $42 \%$ and $53 \%$, respectively, the share of hypoarctic fraction is rather small $(23 \%)$, boreal and polyzonal elements comprise 12 and $15 \%$, respectively. 
Wet communities yielded from 7 (biotope [9]) to 15 ([11]) collected species. Eight species occur rather regularly (found in no less than three biotopes out of five studied ones) but everywhere abundant is only Semljicola alticola (this species share in population of certain biotopes is 28 to 58\%). But Bathyphantes gracilis, B. humilis, Diplocephalus barbiger, Gibothorax tchernovi, Halorates holmgreni, H. spetsbergensis and Semljicola barbiger reach a high abundance only in a few habitats. Arctic species prevail in assemblages of all biotopes (80-97\% of the population), only hypoarcto-montane $B$. humilis is locally abundant among other fractions.

Amderma River Valley. This element of landscape containing a complex of intrazonal specific biotopes we will consider separately because it is of a particular interest. The fact is that the river valleys are the important suppliers of southern faunistic elements into tundra and thus plays an important role in the formation of the fauna of the tundra zone as a whole (see Chernov, Matveeva [2002]; Rybalov [2002]; Tanasevitch, Koponen [2007]; Tanasevitch, Nekhaeva [2016], etc.). All biotopes in the river valley we divided into two types: 1) rather dry rocky banks, stony slopes and stony debris in dried riverbed, and 2) flood-plain habitats willow shrubs, moss and grass communities in depressions. The spider fauna of the river valley as the whole lists 34 species and is represented mainly by species of the Holarctic group, with purely Siberian species comprising less than $21 \%$. The share of arctic fraction $(50 \%)$ is here one of the lowest among all considered types of communities. The same low value of this index was recorded only for the fauna of wetlands but here the share of purely arctic species is $42 \%$ of the fauna, whereas it is only $29 \%$ in the valley fauna. The share of entire arctic fraction is different in these two types of valley biotopes: it is $48 \%$ on dry rocky banks whereas it reaches $57 \%$ in wet flood-plain willow shrubs communities. Among representatives of boreal and hypoarctic fractions the latter are especially diverse (12 species, $35 \%$ of the fauna).

The fauna of mossy willow shrubs communities of the river flood-plain (21 species) contains three specific species found only here. They are two species of hypoarctic fraction: Agyneta similis and Scotinotylus evansi, and a boreo-montane Macrargus multesimus, a typical dweller of boreal forests and southern shrubby tundra, which penetrated here into the subzone of typical tundra along river valley. This species, together with another member of boreal fraction, Allomengea scopigera, belong to specific dominants of this biotope (in total, their share is ca. $22 \%$ of all collected specimens). Almost two thirds of spider population of floodplain willow shrubs communities (64\%) are arctic species, $49 \%$ of them accounted for Semljicola alticola. High abundance of this species and the presence of other arctic elements occurring in wet biotopes of the watershed (Halorates holmgreni, Hilaira incondita, $H$. nivalis, Semljicola barbiger, S. simplex), show resemblance in spider assemblages of these habitats. The group of "southern" species (hypoarctic Bathyphantes humilis, Tmeticus nigriceps, Silometopoides sphagnicola, boreal Leptorhoptrum robustum and polyzonal B. gracilis) is also rather similar, which indirectly indicates quite stable positions of these species in wet biotopes of this region.

Fauna of rocky banks is richer than that of wet willow shrubs habitats in both the number of species (27 vs 21) and the diversity of "southern" elements (14 vs 9). The differences are even more distinct in comparison of this fauna with a similar biotope on the watershed, monadnocks, where the number of found species is twice (13 vs 27 ) and the number of "southern" species is three times lower (5 vs 14). It is evident that just dry bank slopes in the river valley are the place of increased concentration of rare elements of the spider fauna and play the role of refugium for them. Only in this area, on well warmed dry rocky slopes, between stones and on rocks, the following species were found: Alopecosa hirtipes and Erigone tirolensis (both from arctic fraction), as well as Islandiana cristata and Bathyphantes reprobus (both from hypoarctic fraction). Petrophilous Proislandiana pallida, besides rocky river banks, was found only on the watershed monadnocks. Dominants of the population include both arctic species common with spider assemblages of monadnocks, i.e., Agyneta nigripes, Oreoneta leviceps, Halorates holmgreni, and specific for this type of biotopes species: arctic Erigone tirolensis and hypoarctic E. longipalpis, Improphantes complicates and Scotinotylus evansi. In total, the share of hypoarctic species in the population comprises about $35 \%$, that of arctic species is about $58 \%$.

It was mentioned above that southern faunistic elements penetrate into tundra along river valleys. Here, in typical tundra of Amderma, we can see a similar picture. Quite evidently, the river was a channel of expansion of some species of boreal group into typical tundra. Some of boreal elements did not come out of the valley to the watershed, e.g., Macrargus multesimus, while the other species successfully colonized anthropogenic habitats, e.g., Mecynargus paetulus, and/ or wet intrazonal biotopes on the watershed, e.g., Allomengea scopigera and Leptorhoptrum robustum. A species of hypoarctic fraction, Erigone longipalpis, probably also penetrated into typical tundra via the river valley and, besides flood-plain anthropogenic habitats, has broadly colonized also the village territory, where it comprises a considerable part of population of the rubbish heap fauna.

\section{Azonal communities (Azon)}

Sea pebblestone beach. Fauna of this biotope contains 12 species among which there are no specific elements. Species of boreal fraction are lacking here, and elements of arctic fraction comprise $67 \%$. This is a rather high value, only zonal tundra and slopes of hills show higher values (76 and 75\%, respectively). The main part of the spider population (about $73 \%$ ) consists of species from arctic fraction. Most abundant of 
them are species common with monadnocks (Agyneta nigripes, Halorates holmgreni, Oreoneta leviceps), one more species, Erigone arcticola, reaches its high abundance only in these communities. Besides, the dominants include the hypoarcto-montane species Tmeticus nigriceps ( $23 \%$ of the population).

\section{Anthropogenic and anthropogenically changed} tundra communities (Anthro)

Anthropogenic and anthropogenically changed natural communities have long become an integral part of Arctic territories. Similarly to river valleys, these biotopes are also often a kind of "suppliers" of both southern elements to the tundra zone (especially to its northern part) and artic element to more south landscapes, for example to forest-tundra [Tanasevitch, Koponen, 2007; Tanasevitch, Rybalov, 2010, 2015].

To this kind of biotopes, we refer the village territory and two strongly changed natural communities a part of river mouth and a plot of tundra where some economic activity was stopped 20-30 years ago with certain subsequent rehabilitation of environment. In total, 29 species of spiders were collected in anthropogenically changed communities (933 specimens of 2580 collected during the study). Three species appeared to be specific for anthropogenic habitats and have not been found in natural communities; of them, Mecynargus paetulus, was rather abundant, and two other species, Oreoneta uralensis and Dictyna major, were collected in a single specimen. The share of arctic fraction in Anthro is one of the lowest among studied habitats (48\%), but all five polyzonal species are present here.

One of anthropogenically changed areas of tundra is situated on a gentle slope in close vicinity of other undisturbed habitats studied by us. Its vegetation is rather close to natural and seemingly is in the state of rehabilitation. In total, 11 species were collected here; representatives of arctic fraction absolutely dominate in both fauna and population (73 and 97\%, respective1y). At the same time, $77 \%$ of all the specimens belong to Halorates holmgreni, an arcto-alpine species with rather broad topic preferences.

Another anthropogenically changed biotope is located on wet flood-plain at the Amderma River mouth. Similarly to the village itself, it is highly littered with debris and rubbish items. In total, 15 species were collected here, the dominants of the population include dwellers of wet (Erigone arctica palaearctica, Hilaira incondita, Semljicola alticola) and gravelly soil biotopes (commonly occurring Agyneta nigriceps, Bathyphantes gracilis, Tmeticus nigriceps, as well as restricted by the river valley Erigone longipalpis and Improphantes complicatus). The share of arctic fraction species is $52 \%$, that of hypoarctic species - $40 \%$ of the population.

Amderma is virtually abandoned village, most houses are destroyed, yards and roads are overgrown with ruderal vegetation, everywhere there is a lot of debris and rubbish (boards, roofing material, etc.), which are excellent shelters for spiders. Here, in the village, 23 spider species were collected; of them: under rubbish items by hand collecting - 19, sifting and pitfall trapping - 12 species. Except for Dictyna major, the spiders collected in the village lack specific elements found only here. The composition of dominants of the population appeared to be very similar to that of anthropogenically changed wet flood-plain (common dominants Agyneta nigriceps, Bathyphantes gracilis, Erigone arctica palaearctica, E. longipalpis, Hilaira incondita, Semljicola alticola and Tmeticus nigriceps), but it includes also some species with similar topic preferences (among hygrophilous - Masikia indistincta, among petrophilous - Oreoneta leviceps and also Halorates holmgreni).

As it has been already mentioned above, anthropogenic habitats, similarly to the river valley, play a considerable role in the tundra fauna formation. Some hypoarctic and boreal species, which likely penetrated through the river valley, e.g., Erigone longipalpis, Mecynargus paetulus, Improphantes complicatus, and probably had no biological abilities to colonize not only zonal but also intrazonal tundra communities on the watershed, nevertheless successfully realized their reproductive potential just here, in anthropogenic habitats, using debris, remnants of constructions, etc., as secure shelters substantially smoothing the extremal conditions.

\section{Peculiarities of topical preference in repre- sentatives of different latitudinal fractions}

Different species to a different extent explore various habitats of local tundra. Some species colonize almost the entire range of vegetation types; most of them are species of arctic fraction: Halorates holmgreni, Hilaira nivalis, Semljicola alticola, S. barbiger, Xysticus albidus, and only one of them, Bathyphantes humilis, is from hypoarctic fraction. Some species are restricted in distribution by only zonal tundra: these are arctic Arcterigone pilifrons and Mecynargus tundricola. Seven species are restricted in distribution in studied area by the river valley only and do not go beyond: Agyneta similis, Bathyphantes reprobus, Erigone tirolensis, Islandiana cristata, Macrargus multesimus, Scotinotylus evansi and Alopecosa hirtipes. A typical boreal species Mecynargus paetulus and the polyzonal species Dictyna major were found in anthropogenic habitats only.

It should be specially mentioned that some typical arctic species mostly inhabit intrazonal wet biotopes on the watershed or willow shrubs communities in the river valley; for example, Diplocephalus barbiger, Erigone psychrophila, Halorates spetsbergensis, Hilaira proletaria and Semljicola simplex, are absent in zonal tundra and were found in wet intrazonal biotopes only. The share of purely arctic species in intrazonal wetlands is twice higher than in zonal tundra. The river valley also shows a greater degree of association of arctic species with wet habitats: the share of species of 
arctic fraction is higher in wet willow shrubs communities than on dry rocky slopes of the valley and in stony debris (38 vs $30 \%$ of the biotope fauna, respectively).

Concerning the composition of species having high indices of abundance in Amderma vicinities (which considerably forming the species assemblage of certain habitats), it should be noted that representatives of arctic fraction are leading and leave other fractions far behind. Particularly high their share is in the population of spiders on the watershed habitats. Only few species from other latitudinal fractions (i.e., Bathyphantes humilis, B. gracilis, Tmeticus nigriceps, Tubercithorax subarcticus) occur on the watershed and show a relatively high abundance. Limited set of biotopes demonstrating this pattern allows to suggest that local increase in abundance of these species can be a result of anomalously warm weather of the certain summer season.

Most species with high abundance can be rather clearly divided into three groups by their topic preferences:

1) Mesophilous - species with maximum abundance in habitats with humidity close to mean values, e.g., zonal tundra and slopes of hills. They are represented nearly exclusively by species of arctic fraction: Arcterigone pilifrons, Hilaira glacialis, Mecynargus tundricola, Semljicola arcticus, S.barbiger, Alopecosa mutabilis, Xysticus albidus, as well as hypoarctic Tubercithorax subarcticus.

2) Hydrophilous - species with obvious propensity to wet habitats. It is the richest in the number of species group. It is mainly represented by purely arctic species: Diplocephalus barbiger, Erigone arctica palaearctica, Gibothorax tchernovi, Halorates spetsbergensis, Hilaira incondita, H. nivalis, Masikia indistincta, Semljicola alticola). Few hygrophilous boreal elements are associated with the river valley, i.e., Allomengea scopigera and Macrargus multesimus.

3) Petrophilous — dwellers of grounds with high content of gravel/pebble/rubble, stony debris, screes, etc. This group mainly contains species with arctoalpine and hypoarcto-montane distribution: Agyneta nigripes, Erigone arcticola, E. longipalpis, E. tirolensis, Halorates holmgreni, Improphantes complicatus, Islandiana cristata, Oreoneta leviceps, Proislandiana pallida and Tmeticus nigriceps.

Representatives of these three groups differently colonize the disturbed biotopes. The group of petrophilous is especially well represented in anthropogenic habitats, which apparently is due to the presence of many items playing the role of rubble substrate. Besides, the disturbed biotopes occur several hygrophilous species and some of them, i.e., Bathyphantes humilis, Erigone arctica palaearctica, Hilaira incondita and Masikia indistincta are more abundant in anthropogenic habitats than in natural communities. On the contrary, species with highest abundance in zonal tundra are rather poorly and scarcely represented in anthropogenic biotopes.

\section{Peculiarities of distribution of spiders over different layers}

Most spiders of the studied area were collected in the ground/moss layer (pitfall trapping, sifting, collecting under rubbish items), and only a small part was collected in the grass/undershrub layer by net-sweeping. Net-sweeping method resulted in findings of 13 species (Table 2) including 8 species of Linyphiidae with those prevailing species which mainly inhabit the ground/moss layer (an exception - Tenuiphantes nigriventris). On the contrary, other families were represented in the the grass/undershrub layer by quite rare species (except for Xysticus albidus), three of which were collected mainly (Larinioides cornutus) or exceptionally (Dictyna major, Ozyptila arctica) by netsweeping.

Net-sweeping method was used during 11 days. Most time the spiders were collected very few and constantly taken in one place only, i.e., in the village, in wet herb-sedge meadow located in wind shadow of abandoned multistorey building. Exceptions were two days which were not the warmest but sunny and almost calm (Table 2). In these days, spiders were collected not only in the village but also in plots with natural vegetation on the watershed. Most species were collected in habitats with most well developed grass layer, i.e., various meadows, especially associated with relief depressions; one species, Semljicola barbiger, was mainly caught in zonal tundra.

Thus, the results obtained show that the true chortobiont species, as Larinioides cornutus and Dictyna major, are very scanty in the area of study, whereas some moss-dweller spiders (mainly from the genus Semljicola) rise into grass layer only sporadically, in days with especially favourable weather conditions.

\section{Conclusions}

The fauna of spiders of typical tundra in the Amderma vicinities consists of 53 species belonging to 7 families. The fauna is of oligotaxon pattern, its faunistic taxonomical index (FTI) is described by the formula $\operatorname{Lin}(85)-\mathrm{Lyc}(4)-\mathrm{Tho}(4)$ and corresponds to that of other local and regional faunas of typical tundra of Eurasia, though differing by a lower share of Lycosidae.

The fauna can be characterized as Siberian, with high proportion of widely distributed species and a small share of European and East Palearctic elements. The entire share of arctic fraction in the fauna (arctic, arcto-alpine and arcto-montane) comprises $58 \%$, that of hypoarctic fraction (hypoarctic, hypoarcto-montane and hypoarcto-boreo-montane) $-30 \%$, boreal and polyzonal fractions comprised $8 \%$ and $9 \%$, respectively.

In the fauna composition, the main zonal-landscape types of plant communities were distributed as follows: intrazonal (48 species), anthropogenic (29), zonal (17) and azonal (12). 
Zonal tundra prevail in both share of species of the arctic fraction $(76 \%)$ and the share of pure arctic elements $(53 \%)$, species of boreal fraction are completely absent there. The share of species of the arctic fraction in intrazonal biotopes is on the whole considerably lower than in zonal biotopes - 54\%, whereas the share of species of hypoarctic fraction is, on the contrary, much higher ( 29 vs $18 \%$ ); the shares of elements of hypoarctic and boreal fractions are also small $(8 \%$ each). In the species and fraction composition, slopes of hills and zoogenic habitat are most similar to zonal tundra.

The river valley is the source (or refugium) of "southern" elements and thus remarkably increases their share in the fauna of local tundra. Some of these species are restricted in their distribution by the river valley only (7 species), but other species could colonize intrazonal and/or anthropogenic habitats on the watershed where they successfully realized their reproductive potential and formed a marked share in population of some biotopes.

Species of arctic fraction colonized a wider range of types of plant community as compared to species of the other fractions. However, only a small part of them shows an association with zonal tundra, and only two species were recorded only there, i.e., Arcterigone pilifrons and Mecynargus tundricola. Most species of arctic fraction are absent in zonal communities and dwell usually in intrazonal habitats on the watershed and/or in the river valley. The purely arctic species colonize mostly intrazonal wet habitats on the watershed or willow shrubs communities in the river valley; in contrary, the most of arcto-montane and arctic-alpine species are very restricted in wet biotopes but definitely prefer habitats with gravelly soil. Clear topical preferences marked for quite a number of species of the arctic fraction are a specific character of their distribution in studied area only. Many of them inhabit a substantially wider range of biotopes in Siberian tundras.

Species of boreal fraction are absent in most types of plant communities, concentrating in wet intrazonal biotopes, in the river valley, and in anthropogenic habitats.

Three types of population of spiders are clearly distinguished in the composition of dominants in zonal, wet, and petrophite habitats. Spider assemblages of zonal tundra is highly separated from that of other biotopes, it mostly consists of species of the arctic fraction that are common with Siberian tundras, i.e., Arcterigone pilifrons, Hilaira glacialis, Mecynargus tundricola, Semljicola arcticus, Semljicola barbiger and Alopecosa mutabilis. Spider assemblage of wetlands differs in marked variability of the composition of dominants, most common species in all biotopes is Semljicola alticola. On plots with gravelly soil (monadnocks, stony banks and rocky slopes, sea coast), the dominants include Agyneta nigripes, Halorates holmgreni, Oreoneta leviceps and Tmeticus nigripes, the highest share in the population belongs to species with arcto-montane and arcto-alpine distribution.
Species associated with gravelly soil most often occur under rubbish items in anthropogenic and other disturbed habitats, to a lesser extent such biotopes are inhabited by dwellers of wetlands and, very rarely, by those of zonal communities.

Main part of the species (and vast majority of specimens) were collected in the ground/moss layer. Only some rare and scanty in the region species are associated with the grass layer, i.e., Tenuiphantes nigriventris, Larinioides cornutus, Dictyna major and Ozyptila arctica, some common species of the genus Semljicola, i.e., S. alticola and S. barbiger, were recorded in the herbage in some days with favorable (sunny and calm) weather conditions.

ACKNOWLEDGEMENTS. We are greatly indebted to Igor A. Lavrinenko (Botanical Institute of the Russian Academy of Sciences, Saint Petersburg) for the help in geobotanical regionalization and to $\mathrm{Na}$ dezhda B. Leonova (Moscow State University) for the help in identification of mosses and willows. Special thanks go to Dmitry V. Logunov (Manchester, UK) for his helpful comments. The reported study was funded by the Russian Foundation for Basic Research according to the research project \# 15-04-05964 A.

\section{References}

Bulavintsev V.I., Babenko A.B. 1983. [Soil-dwelling invertebrates in the eastern sector of Franz Josef land] // Zool. Zhurn. Vol.62. No.7. P.1114-1116 [in Russian, with English summary].

Chernov Yu.I. 1978. [The structure of the animal population of the Subarctic]. Moscow: Nauka Publ. 165 pp. [in Russian]

Chernov Yu.I., Matveyeva N.V. 2002. [Landscape-zonal distribution of the arctic biota species] // Uspekhi sovrem. biol. Vol. 122. No.1. P.26-45 [in Russian].

Crosby C.R., Bishop S.C. 1928. Revision of the spider genera Erigone, Eperigone and Catabrithorax (Erigoneae) // New York State Museum Bulletin. No.278. P.1-73.

Eskov K.Yu. 1981. [Spiders of the genera Eboria, Latithorax, Rhaebothorax and Typhochrestus (Aranei, Linyphiidae) in the fauna of Siberia] // Zool. zhurn. Vol.60. No.4. P. 496-505 [in Russian, with English summary].

Eskov K.Yu. 1985. [Spiders of the tundra zone of the USSR] // Ovcharenko V.I. (ed.). Fauna i ekologiya paukov SSSR. Trudy Zool. Inst. AN SSSR. Leningrad. Vol.139. P.121-128 [in Russian].

Eskov K.Yu. 1986. On Veles Pakhorukov 1981 and Wubanoides n.gen., two Siberian linyphiid genera (Arachnida: Araneae: Linyphiidae) // Senckenberg. biol. Vol. 67. No.1/3. P. 173182.

Eskov K.Yu. 1987. [New data on spiders of the genus Hilaira (Aranei, Linyphiidae) in the fauna of the USSR] // Zool. Zhurn. Vol.66. No.7. P.1020-1031 [in Russian, with English summary].

Eskov K.Yu. 1988a. The spider genera Savignya Blackwall, Diplocephalus Bertkau and Archaraeoncus Tanasevitch (Aranei, Linyphiidae) in the fauna of Siberia and the Soviet Far East // Folia ent. Hung. Bd.49. P. 13-39.

Eskov K.Yu. 1988b. [Spiders of the genera Mecynargus, Mecynargoides gen.n. and Tubercithorax gen.n. (Aranei, Linyphiidae) in the fauna of the USSR] // Zool. zhurn. Vol.67. No.12. P.1822-1832 [in Russian, with English summary].

Esyunin S.L. 2015. [The spider (Aranei) fauna of the Urals: diversity, structure, typification] // Caucasian Entomological bull. Vol.11. No.2. P.237-257 [in Russian, with English summary]. 
Esyunin S.L., Styopina A.S. 2014. [The fauna and biotopic distribution of the spiders (Aranei) in the southern taiga subzone of the West Siberia] // Vestnik Permskogo Universiteta. Ser. Biologia. No.4. P.24-54 [in Russian].

Gorodkov K.B. 1984. [Ranges types of insects of tundra and forest zones of European Part of U.S.S.R.] // Areas of insects of European Part of U.S.S.R. Atlas. Maps 179-221. Leningrad: Nauka. P. 3-20 [in Russian].

Helsdingen P.J. van 1965. Sexual behaviour of Lepthyphantes leprosus (Ohlert) (Araneida, Linyphiidae), with notes on the function of the genital organs // Zoologische Mededelingen. Vol.41. P.15-42.

Khruleva O.A. 1987. [Invertebrates] // Flora i fauna zapovednikov SSSR: Fauna zapovednika "Ostrov Wrangelya". Moscow. P.636 [in Russian].

Khruleva O.A., Berezin M.V. 2002. Arthropod availability // Willems F., van Turnhout C., van. Kleef H. \& Felix R. (eds.) Breeding birds of Medusa Bay, Taimyr, Russia. Methods for biological monitoring in the Arctic with results of 1998 and 1999, WIWO-report 77, Foundation WIWO, Zeist. P.79-100.

Kulczyński W. 1916. Araneae Sibiriae Occidentalis Arcticae // Mém. Acad. Imp. Sci. Petrograd. Sér.VIII. Cl. phys.-math. Vol.28. No.11. P.1-44, Tab.I-II.

Lavrinenko I.A. 2013. [Geobotanical regionalization of Bolshezemelskaya tundra and adjacent territories] // Geobotanicheskoe kartirovanie. St.-Peterburg. P.74-92 [in Russian].

Marusik Yu.M., Eskov K.Y. 2009. Spiders (Arachnida: Aranei) of the tundra zone of Russia // Golovatch S.I., Makarova O.L., Babenko A.B., Penev L.D. (eds.). Species and communities in extreme environments. Festschrift towards 75th anniversary and a laudatio in honour of Academician Yuri Ivanovich Chernov. Sofia-Moscow: Pensoft Publishers \& KMK Sci. Press. P.131-164.

Marusik Yu.M., Koponen S., Makarova O.L. 2016. A survey of spiders (Araneae) collected on the arctic island Dolgiy $\left(69^{\circ} 12^{\prime} \mathrm{N}\right)$, Barents Sea // Arachnology. Vol.17. No.1. P.10-24.

Mazura N. 2000. 6.6. Spiders // Pechora Delta: Structure and Dynamics of the Pechora Delta Ecosystems (1995-1999). Syktyvkar, Ledystad. P.133-137.

Mazura N.S., Esyunin S.L. 2001. Fauna and biotopic distribution of the spiders (Arachnida: Aranei) in the tundra zone of the North-East of Russian Plain // Arthropoda Selecta. Vol.10. No.1. P.75-81.

Medvedev L.N. 1984. [Quantitive method of zoogeographic analysis of a fauna] // VII Vsesoyuznaya zoogeograficheskaya konferentsiya. Tezisy dokladov. P.211-215 [in Russian].

Nekhaeva A.A., Nekhaev I.O. 2011. On the spider fauna of Bolshoy Aynov Island (Barents Sea) (Arachnida: Aranei) // Arthropoda Selecta. Vol.20. No.4. P.319-322.

Osipov D.V. 2003. [Structure of the spider (Aranei) fauna in southern tundra of western Taimyr] // Zool. zhurn. Vol.82. No.10. P.1266-1270 [in Russian, with English summary].

Pesenko Yu.A. 1982. [Principles and methods of quantitative analysis in faunistic research]. Nauka, Moscow. 286 p. [in Russian].

Pokrovsky S.V. 1904. [Spiders collected by Kanin expedition] // Bull. Rus. Geogr. Obshchestva. Vol.41. No.1. P.297-310 [in Russian].
Rybalov L.B. 2002. [Zonal-landscape change in population of soil invertebrates in Cis-Yenissei region of Middle Siberia and the role of temperature adaptations in zonal distribution of invertebrates] // Russian Entomological Journal. Vol.11. No.1. P.7786 [in Russian, with English summary].

Tanasevitch A.V. 2000. On some Palaearctic species of the spider genus Agyneta Hull, 1911, with description of four new species (Aranei, Linyphiidae) // Arthropoda Selecta. Vol.8. No.3. P.201-213.

Tanasevitch A.V. 1982. [A new genus and species of spiders of the family Linyphiidae (Aranei) from the Bolshezemelskaya tundra] // Zool. zhurn. Vol.61. No.10. P.1501-1508 [in Russian, with English summary].

Tanasevitch A.V. 1984a. [New and little-known spiders of the family Linyphiidae (Aranei) from the Bolshezemelskaya tundra] // Zool. zhurn. Vol.63. No.3. P.382-391 [in Russian, with English summary].

Tanasevitch A.V. 1984b. [New species of spiders of the genus Agyneta Hull, 1911 (Aranei, Linyphiidae) from Siberia and Middle Asia] // Nauchn. dokl. vyssh. shk. Biol. nauki. No.5. P.47-53 [in Russian].

Tanasevitch A.V. 1985. [To the study of the spiders (Aranei) of the Polar Urals] // Ovcharenko V.I. (ed.). Fauna i ekol. paukov SSSR. Trudy Zool. inst. AN SSSR. T.139. P.52-62 [in Russian].

Tanasevitch A.V. 2017a. New species and new records of linyphiid spiders from the Indo-Malayan Region (Araneae: Linyphiidae) // Zootaxa. Vol.4227. No.3. P.325-346.

Tanasevitch A.V. 2017b. New records of spiders (Aranei) from the Russian Arctic // Arthropoda Selecta. Vol.26. No.1. P.77-82.

Tanasevitch A.V. 2017c. Spiders (Aranei) of the Novaya Zemlya Archipelago and the Vaygach Island, Russia // Arthropoda Selecta. Vol.26. No.2. P.145-153.

Tanasevitch A.V., Eskov K.Y. 1987. [Spiders of the genus Lepthyphantes (Aranei, Linyphiidae) in the Siberian and Far Eastern fauna] // Zool. zhurn. Vol.66. No.2. P.185-197 [in Russian, with English summary].

Tanasevitch A.V., Koponen S. 2007. Spiders of the southern tundra in the Russian Plain // Arthropoda Selecta. Vol.15. No.4. P.295-345.

Tanasevitch A.V., Nekhaeva A.A. 2016. Spiders (Aranei) of the Kharaulakh Mountains and the Lena River lower reaches, northern Yakutia, Russia // Arthropoda Selecta. Vol.25. No.3. P.307326.

Tanasevitch A.V., Rybalov L.B. 2010. On spiders from tundra zone of Kola Peninsula, Russia (Arachnida, Aranei) // Arthropoda Selecta. Vol.19. No.1. P.41-56.

Tanasevitch A.V., Rybalov L.B. 2015. Fauna and distribution of spiders (Aranei) of the arctic tundra in northern Yamal Peninsula, Russia // Arthropoda Selecta. Vol.24. No.2. P.215-230.

Taskaeva A.A., Kudrin A.A., Konakova T.N., Kolesnikova A.A. 2015. [Diversity of soil invertebrates in ecosystems near the Padimeyskie lakes in the Bolshezemelskaya tundra] // Eurasian Entomological Journal. Vol.14. No.5. P.480-488 [in Russian, with English summary].

Yurtsev B.A., Koroleva T.M., Petrovsky V.V., Polozova T.G., Zhukova P.G., Katenin A.E. 2010. [Checklist of Flora of the Chukotkan Tundra]. VVM. St. Petersburg. 628 pp. [in Russian].

Responsible editor K.G. Mikhailov 


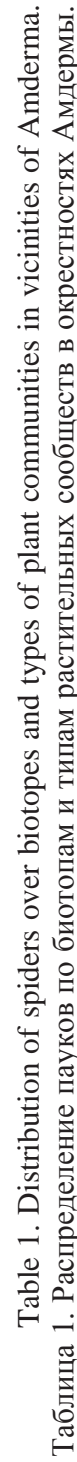

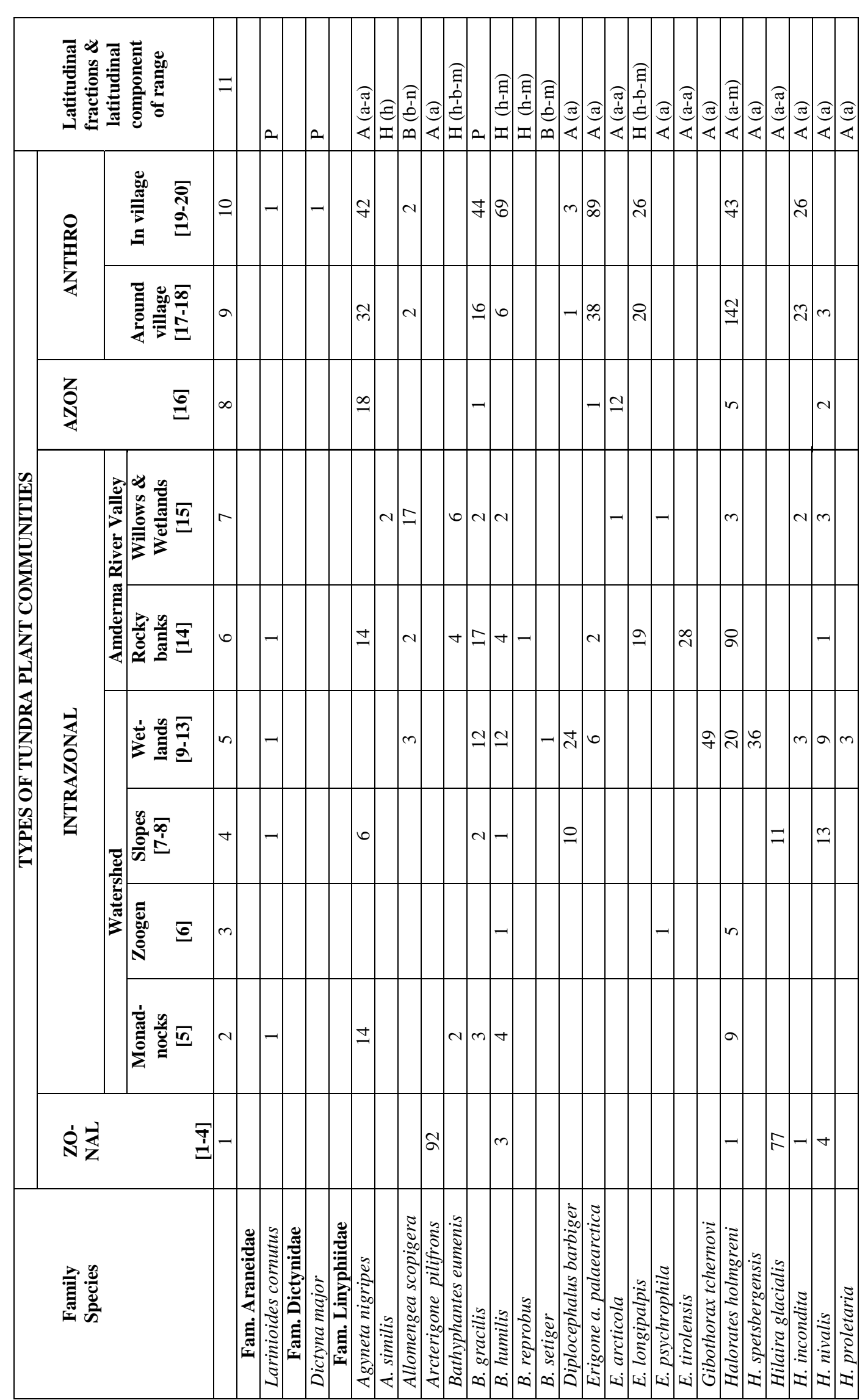




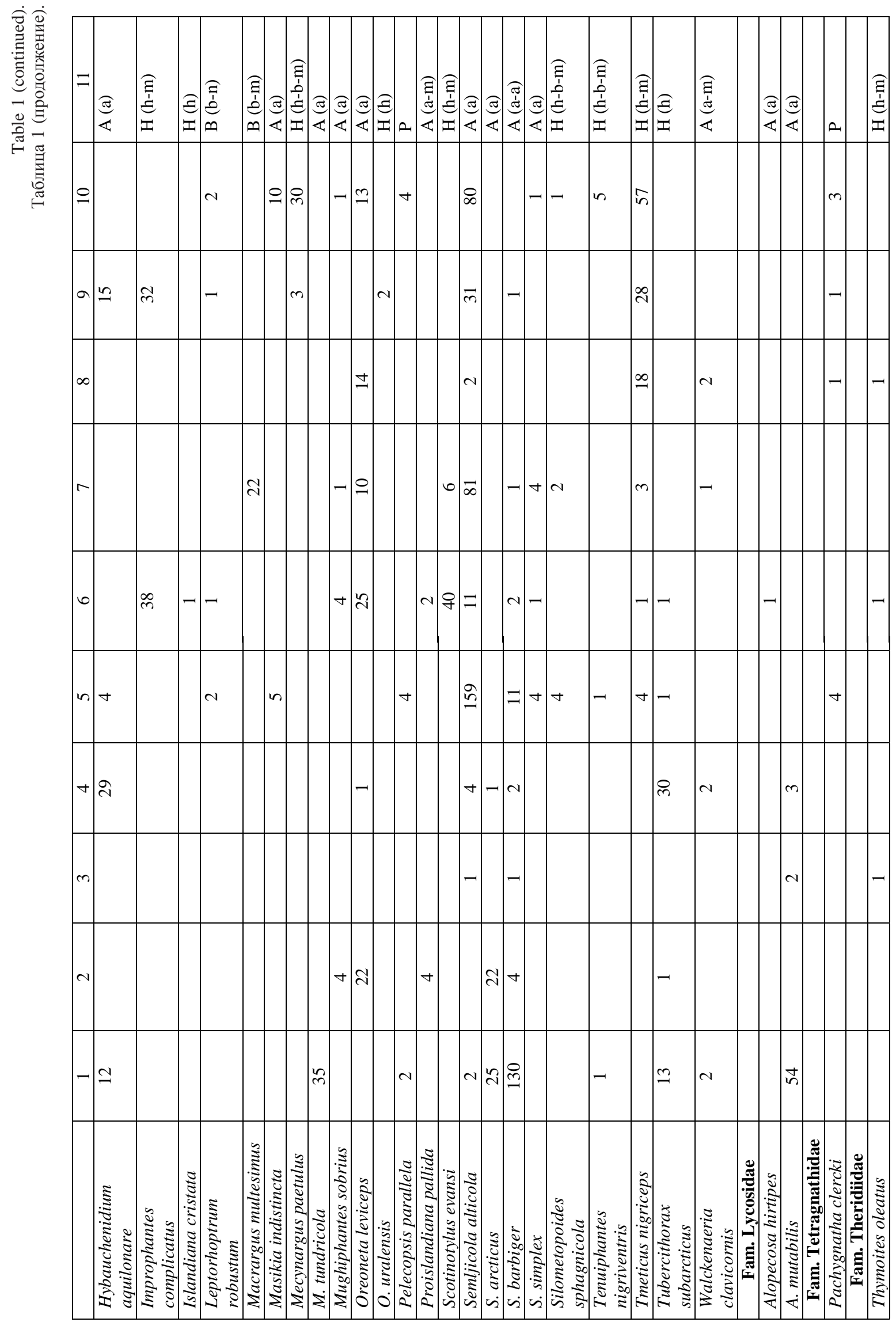




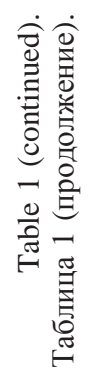

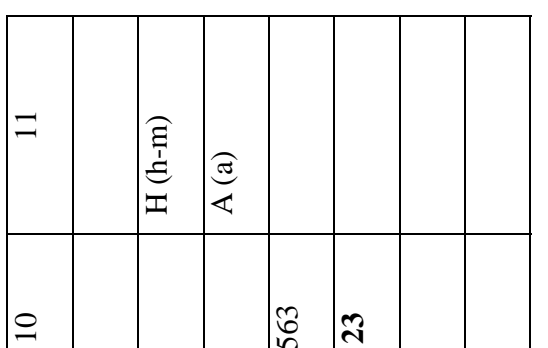

잉

央

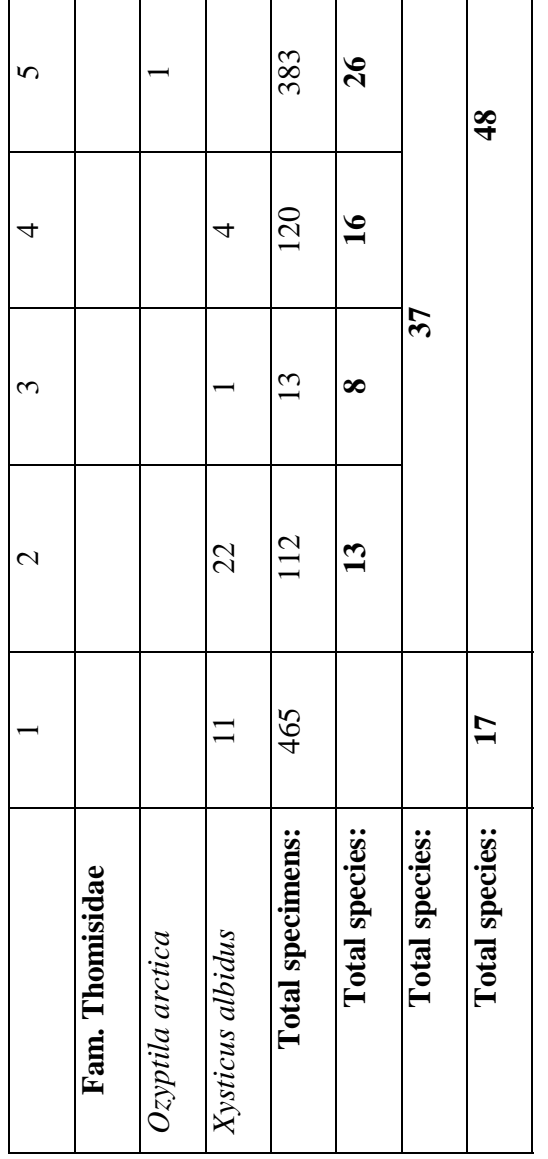

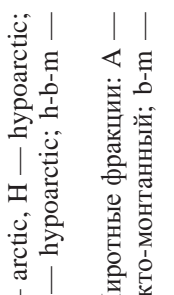

I. 晋量

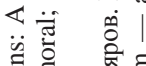

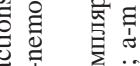

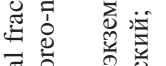

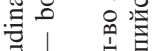

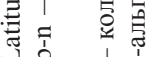

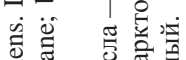

坖氜

के

월

สิ

ญे है।

矛苛

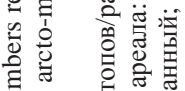

है 잉 च

苍志

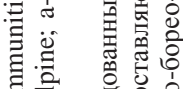

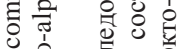

芯递哥要

क |

원 인

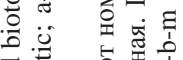

氖焉者

范 |

范

हี

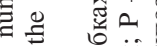

范。总岛

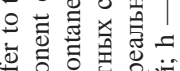

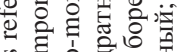

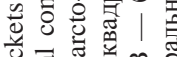

중

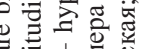

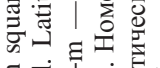

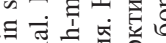

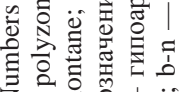

之 |

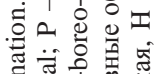

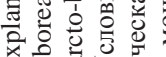

文| 
Table 2. Spiders collected by net-sweeping in days with different weather conditions.

Таблица 2. Пауки, собранные в укосах в дни с разными погодными условиями.

\begin{tabular}{|c|c|c|c|}
\hline Species & $\begin{array}{l}\text { 30.VII., sunny, slight } \\
\text { wind, 13-15 }{ }^{\circ} \mathrm{C}\end{array}$ & $\begin{array}{c}\text { 2.VIII., sunny, calm, } \\
10-12{ }^{\circ} \mathrm{C}\end{array}$ & Other days \\
\hline Bathyphantes humilis & - & $1 \bigcirc^{7}[12]$ & - \\
\hline Mughiphantes sobrius & - & - & 1 juv. [19] \\
\hline Pelecopsis parallela & - & 2 우우 $[11,12]$ & - \\
\hline Semljicola alticola & 1 ㅇ [19] & $290^{7} \bigcirc^{7} \&$ of $[12,11,8]$ & - \\
\hline Semljicola barbiger & $5 \circ[3]$ & $14 \sigma^{7} \sigma^{7} \&$ \& $[2,3,6,7,9]$ & - \\
\hline Semljicola simplex & - & $1 \%[12]$ & - \\
\hline Tenuiphantes nigriventris & $5 \bigcirc^{7} \bigcirc^{7} \&$ \& $+9[19,3]$ & $10^{7}[9]$ & - \\
\hline Tmeticus nigripes & -- & - & $1 \lesssim[19]$ \\
\hline Larinioides cornutus & - & - & 3 juv. [19] \\
\hline Thymoites oleatus & - & 1 juv. [6] & - \\
\hline Ozyptila arctica & - & - & 1 우 [10] \\
\hline Xysticus albidus & - & - & 2 juv. (river valley*) \\
\hline Dictyna major & $19[19]$ & - & - \\
\hline $\begin{array}{r}\text { Total net-sweeping } \\
\text { (net-sweeping samples): }\end{array}$ & $6(1000)$ & $8(1575)$ & $38(7725)$ \\
\hline Total specimens collected: & 12 & 49 & 8 \\
\hline
\end{tabular}

Explanation. In square brackets are given the numbers of biotopes where the spiders were collected (in decreasing order as concerning the number of collected specimens). * - net-sweeping in sandy-pebble river flood-plain with a sparse grass cover.

Примечание. В квадратных скобках приведены номера биотопов, в которых собраны пауки (расположенные в порядке убывания кол-во собранных экз.). * - укосы на песчано-галечниковой пойме с разреженным разнотравно-злаковым покров. 